\title{
Abstracts of the UNICAMP Academic Medical Congress (COMAU), 2019
}

\section{Basic Science}

01. IN VITRO AND IN VIVO EFFECT OF GALLIC ACID ON PROLIFERATION AND APOPTOSIS OF ACUTE MYELOID LEUKEMIA CELLS

João Vitor de Paula Souza Luz, Sara Teresinha Olalla Saad, Marisa Claudia Alvarez de Prax

Universidade Estadual de Campinas - UNICAMP

Acute Myeloid Leukemia (AML) is a systemic neoplasm that is formed into the bone marrow and is responsible for $90 \%$ of all cases of Acute Leukemia in adults, it is more common in people over 65 years, which have a worse prognosis. Chemotherapy is used to treat AML and it is possible to achieve survival over than 5 years in about $20 \%$ of cases, but there are elderly people who cannot tolerate aggressive therapy due to the high toxicity of chemotherapy agents. Bone marrow transplantation increases survival up to $50 \%$, but older patients are often ineligible for this (kind of) treatment Many chemotherapy drugs used in the treatment of neoplasms, including AML, are obtained from plants. Galic acid (GA) is a natural product that can bring benefits for the treatment, including preventive properties and low cost of production. It is described as an antioxidant with epigenetic modulation properties, which induces cell death by apoptosis, antiproliferative activity and inhibition of angiogenesis against tumor cells. Moreover, GA has low cytotoxicity, which makes it more interesting for the treatment of neoplasms, even though studies in vivo in AML models are still lacking. The aim of this study is to analyze the in vitro and in vivo effects of GA in AML models. For this purpose, myeloid leukemia cell lines and animals with xenogenic implants of leukemic lineages were used. CA has been shown to reduce cell viability in human leukemic cell lines (HL6O and U937), to induce cell apoptosis, to inhibit tumor growth in xenographic grafts in in vivo assays, to increase intracellular concentration of Reactive Oxygen Species, to increase the expression of the proapoptotic Bax protein, and also was shown, by parcial results, that drug's influence could induce $\mathrm{G}_{1}$ arrest while reducing $\mathrm{C}_{2}$ cell population. It is concluded that $\mathrm{CA}$ has effects on proliferation and apoptosis, with promising potential in combined chemotherapy for the treatment of AML.

02.

ASSESSMENT OF THE IMPORTANCE OF CONTINUING EDUCATION IN CARDIAC ANATOMY FOR TRANSSEPTAL PUNCTURE PROCEDURE

Isabele Cristina Targon Fukumaru, Silvio Giopatto, Caroline de Souza Silva, Leslie Cristina Pinto Levy, Cândida Luiza Tonizza de Carvalho Pontifícia Universidade Católica de Campinas

In the last decade, transseptal puncture (TSP) has gained great relevance in interventional and hemodynamic cardiology. In this context, the retrieval of knowledge about the fossa ovalis anatomic relationships for the procedure is essential. Thus a Hands on - Anatomy and transseptal puncture in animal model was performed at the Congress SOLACI-SBHCl 2019, focusing on the anatomy of the fossa ovalis, its portions, its anatomic relationships and association with the structural cardiac procedures performed through TSP. Approximately $66 \%$ of the participants aimed, in the training, the understanding of anatomical relations and after the training $53.3 \%$ of the participants had an understanding "beyond expectations" and $46.7 \%$ had a "good understanding" about those relations. Therefore, assessing the main expectation of cardiologists as being the understanding of cardiac anatomical relationships and considering the current brazilian scenario of interventional cardiology with a growing number of procedures using TSP can be concluded a need for continuing education in cardiac anatomy. In addition, the success of using animal model in the teaching techniques, despite not used very often on Brazil, has show a new path for the enhancement on the knowledge of fossa ovalis anatomical relationships.

Key-words: transseptal puncture, fossa ovalis, continuing education, cardiac anatomy

03. EVALUATION OF PLASMA BIOMARKERS OF ENDOTHELIAL FUNCTION IN MIDDLE-AGED MEN AND WOMEN WITH TYPE 2 DIABETES

João Felipe Oliveira Santos, Maria Andreia Delbin, Aline Pincerato Jarrete

Universidade Estadual de Campinas - UNICAMP

The aim of the study was to evaluate circulating endothelial function biomarkers in middle-aged men and women ( $45-65$ years) with type 2 diabetes. Interviews, anthropometric measurements, and blood samples were collected from 58 volunteers, which were divided into 4 groups. They were: control men $(\mathrm{HC}, \mathrm{n}=15)$, diabetic men ( $\mathrm{HD}, \mathrm{n}=13$ ), control women $(M C=16)$ and diabetic women $(M D, n=14)$. Subsequently, glucose, triglycerides (TC), LDL-C, HDL-C, VLDL-C, non-HDL-C, insulin, HbA1C, creatinine LDL-ox and ADMA were measured. For LDL-ox and ADMA dosage, ELISA immunoenzymatic tests were performed. No differences were observed in TG, HDL-C, VLDL-C insulin, LDL-ox and ADMA concentrations. However, significant difference was observed in the BMI, AC and WHR parameters, especially the MD compared to the MC group, and also the LDL-C and TC levels, which were lower in the MD compared to the MC group. Regarding the glycemic profile, a significant increase in the blood glucose and $\mathrm{HbA} 1 \mathrm{C}$ concentration of the diabetic groups was observed in relation to the control The ratio LDLox / LDL-C and LDLOX / HDLC was investigated, since it is related to higher risk of cardiovascular events at high levels. It was noted that the MD group had a high LDLox / LDL-C ratio compared to the MC group. Further studies are needed to corroborate the data found in this study and genderspecific differences.

04. THE CD90/Thy1 IN TRIPLE NEGATIVE BREAST CANCER: ASSOCIATIONS BY BIOINFORMATICS AND SYSTEMS BIOLOGY APPROACH BETWEEN DYSREGULATED GENES AND SIGNALING PATHWAYS

Marco Lázaro de Sousa Batista, Aline Ramos Maia Lobba; Mari Cleide Sogayar; Ana Claudia Oliveira Carreira; Milton Yutaka NishiyamaJunior

Universidade de São Paulo - USP

Breast carcinoma is the most frequently diagnosed type of cancer among women, with the ductal-invasive triple-negative being the most aggressive, displaying the worst prognosis. Due to the lack of molecular targets, the treatment of patients presenting this phenotype becomes a great challenge, requiring more extensive studies to better understand the biology of this tumor type for the development of new therapeutic strategies. These studies have been carried out by the NUCEL group of researchers in an attempt to find new molecular targets of clinical interest. To this end the CD9o stem cell marker was identified by Dr. Aline Maia Lobba as being a promising target in breast cancer, since it was associated with the poor prognosis of patients and with several cellular processes which lead to malignant transformation, such as: morphological alteration, epithelialmesenchymal transition, increased cell proliferation, invasiveness, 
metastasis and activation of the EGFR pathway. More than $78 \%$ of triplenegative breast cancer cases show overexpression of EGFR, allowing us to study their components and attempt to identify a potential therapeutic target. Therefore, understanding of the CDgo signaling pathway and its possible relationship with the EGFR pathway should be an interesting approach towards the molecular basis of basal-like mammary tumorigenesis. Therefore, the central objective of this project is elucidation of the $C D 90$ signaling pathway and its relationship to the EGFR pathway in regulation of the downstream gene expression process. This project is being developed through a multidisciplinary approach involving Systems Biology, combined with conventional technologies of Cellular and Molecular Biology for validation of the results obtained through mathematical and Bioinformatics analysis. The knowledge generated in this work has contributed to a better understanding of the pathways and genes involved in the tumor malignancy process, as well as the methodological improvement of the total transcriptomics for the better biological understanding of $\mathrm{CD} 90$.

Key-words: Breast Cancer, EGFR, CD90, Transcriptome, Bioinformatics, Systems Biology.

05. IMMUNOEXPRESSION OF APE-1 AND XRCC1 PROTEINS IN ORAL SQUAMOUS CELL CARCINOMAS

Ian Siqueira Araújo Câmara, Myrna Barbosa Comes, André Maia Ribeiro, Gabriela Tomazini Rodrigues Pereira Amorim, Luara Costa Amorim, Rebeca Mendes Peres UNICEPLAC

Oral squamous cell carcinoma (OSCC) results from the occurrence of cellular events caused by mutations associated with carcinogenic agents, which may lead to the losso control of cell proliferation. OSCC has multifactorial causes, including both extrinsic and intrinsic factors. The study of the proteins involved in the DNA repair mechanism is important for cancer research, since repair gene-related mutations may be a starting point for tumorigenesis chemotherapeutic resistance in malignant cells. Therefore, understanding the role of AP- apurinic/apyrimidinic endonucleases (APE-1) proteins and the X-ray repair cross-complementing group 1 (XRCC 1 ) involved in the repair may help understanding this carcinogenesis. Objective: To review the literature on the expression of repair proteins $A P E-1$ and XRCC 1 , associating them with OSCC and other neoplasms. Methods: A literature review was performed in the Medline, Scielo and Pubmed databases. Articles from 2005 to 2018 were used, excluding that do not address the expression of APE-1 and XRCC 1 proteins as their main theme. Results: APE1 overexpression was evidenced in different organs neoplasms, and correlated with their malignancy. This proteins expression in the OSCC, regarding clinical staging, was high in both early and late stages, related to the carcinoma degree lesion. In addition, in relation to the $\mathrm{XRCC}_{1}$ protein was found an underregulation in several neoplasias as in the carcinogenesis in the gastrointestinal tract and bladder carcinoma. XRCC 1 expression was low in advanced stage groups in the patients with OSCC. Moreover, XRCC1 rates were shown to be higher in healthy tissue cells than those that were involved in the gastric carcinoma process. Regarding a possible analysis between APE-1 and XRCC 1 proteins, there was no significant correlation of their expression together in cancer cells. Conclusion: The APE-1 and XRCC 1 proteins, according to the research results, indicates a significant participation in the OSCC initiation and progression, but these proteins immunoexpression are not significantly associated with the clinical and prognostic parameters in the OSCC cases.

Keyword: Immunoexpression, APE-1, XRCC1 and OSCC

\section{NEUROSTEROIDS AS BIOMARKERS OF NEUROGENESIS}

Gabriel Antonio Roberto, Renato Madlum, Carolina Magalhães Britto União Das Faculdades Dos Grandes Lagos

Neurosteroids are hormones synthesized by brain cells acting during neurodevelopment, from the early stages of the fetus until adolescence and their action depends on appropriate physiological concentrations. Consequently, secondary mechanisms such as neuroprotection, regulation and organization of brain function are also inherent in these substances. However, psychopathological processes such as schizophrenia, epilepsy, depression, eating disorders, aggressive behavior, Autistic spectrum Disorder (ADD), Attention Deficit Hyperactivity Disorder (ADHD) may have a direct correlation with the change in neurosteroid production. Objectives: To attribute to neuro-steroids the function of biomarkers of dysgenesis and neurological disorders, since in this literary review we note the involvement of these steroids in the development of the Central Nervous System (CNS) and mental disorders in children and adolescents. Methods: Systematic study based on the review of journals extracted from the PubMed Database using the terms allopregnanolone, placenta, neurosteroids, having as inclusion criteria the clinical relevance described in the literature. Results and Discussion: Therefore, adequate physiological levels protect the developing neural system from eventual recurrent insults, as well as contribute to the regulation of brain organization and function. Thus, the complex interaction between these neuroactive steroids with environmental, genetic and neurodevelopmental factors in children and adolescents undoubtedly has intrinsic correlation in the clinical presentation of these pathophysiology. Conclusion: Allopregnanolone, produced by the placenta in high concentrations from progesterone in late pregnancy, is an important neurosteroid and essential for brain maturation during extrauterine life. Between this and that, interruptions in the adequate supply of this hormone may alter neurogenesis in critical areas related to social cognition and motor coordination, such as cerebellar white matter. In addition, sudden drops in allopregnanolone supply may result in premature births, a critical risk factor for the onset of Autistic Spectrum Disorder (ASD). Finally, neurostteroids also have therapeutic potential in the treatment of various diseases, such as schizophrenia, depression, aggressive behavior, ADHD and autism. Therefore, the use of these placental or brain biomarkers can undoubtedly be used as tools for hormone supplementation-based treatment in the fetal period.

\section{FIRST AID TRAINING FOR PUBLIC EDUCATION NETWORK STUDENTS}

Leticia Cristina Francisco, Joaquim Simões Neto, Gabriela Acurcio

Barbosa, Eduardo Sbroggio, Karin Hayashi, Marilia Amelotti

Pontifícia Universidade Católica de Campinas - PUC

The present work aims to contribute to the qualification of students from Public schools in Campinas guiding them to carry out the initial care to the common medical emergencies such as choking and cardiopulmonary arrest while await appropriate technical assistance. It is also considered of great importance to preparing the population to perform first aid in situations of emergencies, in which time is a key factor for good prognosis while awaiting specific care from health professionals. Therefore, the course was applied to students starting with theoretical classes that addressed medical emergencies on adults, adolescents and children in situations of sudden illness, choking and seizures, fainting and hypoglycemia, as well as minor home accidents. In the practical classes, the participants were oriented medical students on how to proceed in each situation and after the orientations performed specific first-aid maneuvers on puppets suitable for this purpose. In addition, it was emphasized throughout the course lessons taught in theoretical class, ie how to perform a resuscitation cardiopulmonary bypass in adults and babies, as well as disengagement maneuvers in both. Two evaluations were performed to measure the degree of theoretical and students' practice. Finally, it was noted that there was a significant improvement in students' performance on the second test, which indicates that the course was effective and provided appropriate learning and training for participants.

\section{EFFECTS OF PREOPERATIVE RESPIRATORY MUSCLE TRAINING ON HEART} SURGERY: REVIEW

Isadora Lenzi Veronezi, Priscila Moraes Guido, Lívia Ravazzi

Centro Universitário Nossa Senhora do Patrocínio - CEUNSP

To evaluate the effects of inspiratory muscle training (IMT) applied in the preoperative period of cardiac surgery in adult patients as a way to prevent postoperative pulmonary complications. Methods: A review study was performed using the following databases: PubMed, PEDro, Scielo, LILACS and Cochrane. All types of studies addressing the effects of inspiratory muscle training in the preoperative period of cardiac surgery and meeting the inclusion criteria initially established were included. Results: The full search led to the identification of 670 articles, of which 34 studies were considered potentially relevant. After their full reading, only 13 met the eligibility criteria. Study results Shakouri et al (13) show that the hospitalization time of the $\mathrm{Cl}(45.9 \pm 17.9 \mathrm{~h})$ was shorter when compared to the CC $(69.9 \pm 26.3 \mathrm{~h})$, the mechanical ventilation also showed a difference between the first (10.6 $\pm 3.8 \mathrm{~h})$ and the second group $(17.2 \pm 4.9 \mathrm{~h})$. Postoperative pulmonary complication rates decreased, forced vital capacity (FVC) improved $(95 \% \mathrm{Cl}$ 1.3 to 8.7$)$ and peak expiratory flow (PEF) (95\% Cl: 1.98 to 9.4). Respiratory muscle strength and MIP and MEP values were significantly higher in the group receiving IMT with the $40 \%$ MIP Threshold device observed in the longitudinal study by Sobrinho et al (14). Conclusion: IMT can be effective in reducing postoperative complication rates and length of hospital stay, 
thus accelerating patient recovery. However, further studies are needed to reinforce the evidence described and standardize the approach to training.

Keywords: Heart Surgery, Respiratory Muscle Training, Respiratory Rehabilitation

09.

MELATONIN ACTION AGAINST ISCHEMIA-REPERFUSION INJURY IN CARDIAC TISSUE - A REVIEW

Caroline De Souza Silva, Celene Fernandes Bernardes, Emily Ayumi Kimoto

Pontifícia Universidade Católica de Campinas - PUC

Melatonin is classically known as the pineal gland hormone. However recent studies indicate a cardioprotective action of melatonin in cardiac ischemia-reperfusion (IRI) injury. Myocardial ischemia is the major cause of sudden death worldwide and there are important indicatives of melatonin protective effects. In this review, experimental studies using rats and cells in vitro ( $\mathrm{H}_{9 \mathrm{C} 2}$ cells) with melatonin treatment in the context of IRI were used. The results reveal that melatonin decreases autophagy in cardiac microvasculature endothelial cells by ULK 1 activation and mTOR inhibition. In studies performed with $\mathrm{H}_{9 \mathrm{C} 2}$ cells, melatonin acts via ERK 1 inducing high SERCA2 expression and low IP $3 \mathrm{R}$ expression, this situation results in cardiomyocyte preservation. In addition, melatonin acts on the Nrf signaling pathway, the factor targets the nucleus causing antioxidant and antiapoptosis effects. In mitochondria of H9c2 cells, melatonin increased Sirt3 expression, on the other hand, the IRI decreased Sirt3 expression; consequently, there are less oxidative stress and less apoptosis. The Sirt3 expression also increases the expression of manganese superoxide dismutase, which maintains mitochondria homeostasis. Futhermore, melatonin induces mitochondrial fission repression, autophagy repression and stimulation of mitochondrial fusion by AMKP activity in endothelia cells. In addition, melatonin is responsible by OPA-1 overexpression, contributing to mitochondrial homeostasis. Thus, melatonin has an important protective role in IRI, acting on endothelial cells and cardiomyocytes, mainly reducing oxidative stress. This review shows how these processes occurs.

Key-words: melatonin, mitochondria, oxidative stress, ischemia-reperfusion injury.

\section{EVALUATION OF RESPIRATORY CONDITIONS IN STROKE}

Nataly Cristina Sá de Jesus, Priscila Moraes Guido, Myllena Pintor Parra

Centro Universitário Nossa Senhora do Patrocínio - CEUNSP

Stroke is a neurological event that can lead to functional impairment, including respiratory. Objective: This study aims to analyze the respiratory conditions of stroke patients, assessing respiratory muscle strength by measuring maximal inspiratory pressure (MIP) and maximal expiratory pressure (MEP), body mass index (BMI) and capacity vital force (FVC), the acquired data evaluated possible respiratory complications of the patients. Methods: This is a descriptive cross-sectional study, carried out at Centro Universitário Nossa Senhora do Patrocínio (CEUNSP), Irmã Anna de São Jose Camargo Barros clinic. 25 patients between 35 and 89 age with a diagnosis of ischemic and / or hemorrhagic stroke were evaluated for the vital capacity forced by spirometry and MIP and MEP by manovacuometry and BMI by weight and height. Results: of the 19 patients evaluated, $84 \%$ of patients had decreased MEP and $92 \%$ decreased FVC. Regarding BMI, there was an average of $29.43( \pm 5,915)$ characterizing overweight in $40 \%$ of patients. Discussion: In our study, we found some changes consistent with other studies, which found a decrease in FVC, MIP and MEP, which may be due to weakness of intercostal muscles, changing inspiration and decreasing lung expansion. Regarding the high BMI, studies report to be related as risk for stroke and low FVC values have relationships with low MIP and MEP values and high BMI index. Conclusion: We observed that there are changes in the respiratory system in patients with stroke and in relation to the evaluated data, showed a decrease in FVC and PEmax that may impact the quality of life and social interaction of stroke patients.

Keywords: Stroke, Respiratory Muscles, Maximal Respiratory Pressures, Spirometry, Body mass index, Respiratory System
11. EVALUATION OF CYTOKINE PRODUCTION IN THE ORAL FLUID (SALIVA) OF PATIENTS WITH CONTROLLED ASTHMA AND DIFFICULT CONTROL ASTHMA

Raíssa Alves Jorge, Ronei Luciano Mamoni, Daniela de Queiroz Santana, Isabela Beraldi Eguni, Ana Lúcia Bergamasco Calastri, Eduardo Vieira Ponte

Faculdade de Medicina de Jundiaí

Asthma is a chronic inflammatory disease characterized by inflammation of the airways, leading to its obstruction. It is a disease of high global prevalence that affects all age groups and presents high rates of morbidity and mortality. In general, this pathology can be classified into two main types, based on the immunopathological mechanisms that lead to its development: atopic asthma and non-atopic asthma. Atopic asthma is characterized by a type I hypersensitivity reaction initiated by the exposure to allergens, which induce the release of IL-33 and TSLP, the differentiation of Th2 and The lymphocytes (producing IL-4, IL-5, IL-13, and IL-9), the IgE production and activation of mast cells and subsequent chemoattraction and activation of eosinophils. Non-atopic asthma is usually associated with exposure to lung infections and pollutants that initiate a chronic inflammatory process with the active participation of Th17 and Th22 lymphocytes (IL17 and IL-22 producers) and the activation and chemoattraction of neutrophils. After activation, these different cell types will be responsible for the production of enzymes, reactive oxygen species (ROS) and inflammatory cytokines that will promote the tissue remodeling responsible for the airway obstruction. Despite the advances in the knowledge of the processes involved in the development of asthma, there are several mechanisms to be better understood especially those related to the differences observed in response to the available treatments. As mentioned, the different cytokines produced during the immune response play a fundamental role in the development of asthma and, therefore, a better understanding of their participation in the evolution of the disease can help in the therapy adopted. Evaluation of cytokine levels usually is done in the serum or plasma of patients, but it is not always possible to use this method, since it is invasive and requires trained persons to collect the blood. The aim of this study is to evaluate the production of some cytokines involved in the development of asthma in saliva samples from patients with controlled asthma and uncontrolled asthma.

Keywords: controlled asthma, uncontrolled asthma, cytokines, saliva.

\section{THE BENEFITS OF FUNCTIONAL FOODS IN VASCULAR DISEASES}

Marília Leal Escobar, Lúcio Fábio Caldas Ferraz, Rebeca Funck Colucci, Ana Victória Haddad Universidade São Francisco

Functional foods, also called nutraceuticals, are characterized as norma human diet foods that, in addition to presenting their basic nutritional benefits, are also those that promote physiological benefits to the body and capable of reducing the risks of chronic diseases. An adequate diet with such foods may be related to the prevention of vascular diseases, as well as assisting the recovery of patients undergoing vascular surgical procedures. The present study aims to analyze functional foods and their influence on vascular diseases, as well as expose the importance of proper orientation of such compounds. Functional foods are currently widely studied because they include numerous substances capable of promoting metabolic and physiological effects beneficial to health. Given this, their relationships with the vascular system were studied and it was found that they may have direct and indirect influences on the protection and treatment of vascular diseases. Directly, these components act as antioxidants and anti-inflammatories in the blood vessels. Indirectly, they bring benefits to the cardiac, renal and endocrinological systems, in order to reduce the incidence of metabolic diseases that will result in vascular damage. Concluding, it is noted that functional foods have direct benefits in the area of angiology and vascular surgery, since they have positive effects on the body's homeostasis, such as antioxidant and antiinflammatory action of the vessels. However, it should be noted that the indiscriminate and excessive use of these components can cause health damage too. Therefore it is important to guide the proper diet in order to avoid side effects and toxicity to the patient.

Keywords: Functional Food; Vascular Diseases; Atherosclerosis; Antioxidants; Anti-Inflammatory Agents. 
13.

\section{ANALYSIS OF THE MORPHOLOGICAL AND IMMUNOHISTOCHEMICAL PROFILE OF PATIENTS WITH ACUTE REJECTION AFTER LIVER TRANSPLANTATION AND CORRELATION WITH THE PROGNOSIS}

Milena Stenico, Larissa Bastos Eloy da Costa

Universidade Estadual de Campinas - UNICAMP

BACKGROUND: Acute rejection in the hepatic graft occurs in days, months of years after transplantation and the severe form has been associated with a humoral component, which does not yet present well-defined histological findings. This study aims to understand the mechanisms of acute rejection and clinical-pathological behavior, in order to provide relevant information for prognosis and conduct. METHODS: Retrospective, longitudinal and investigative study. Selection of acute rejection cases between 2010 and 2015, with histological review, immunohistochemical reaction for $\mathrm{C}_{4} \mathrm{~d}$ and clinical-laboratory correlation. RESULTS: 238 transplanted and $16.7 \%$ with acute rejection. Among these, $68.4 \%$ were male, $89.2 \%$ were older than 40 years and $32.5 \%$ had hepatocellular carcinoma. The average amounts of ALT, AST, alkaline phosphatase and bilirubin were 222, 419, 396 e 14.1 (mg/dL), respectively. Significant correlation between higher rates of rejection and endothelitis, aggression to the ductal epithelium, plasmocytes, canalicular cholestasis, portal edema, interface activity, fibrosis, lobular necrosis and total bilirubin levels. Significant difference between death and higher levels of bilirubin and lower frequency of interface activity. Diffuse C4d expression was observed in 3 cases, focal expression in 7 and 5 cases did not express this marker. CONCLUSIONS: Liver graft rejection is less common than other solid organs, but some patients progress unfavorably. Endothelitis and ductal aggression are described in acute cell rejection, but the significant correlation between plasmocytes and higher rejection rates may favor the presence of humoral component. The association between higher bilirubin rates and death may be related to slow cholangitis in the context of shock. c4d expression may contribute to the diagnosis of acute antibody-mediated rejection and guide a more specific treatment.

14. LEISHMANICIDAL EFFECT OF VERNONIA POLYANTHES LESS. PHYTOCHEMICAL AND BIOLOGICAL CHARACTERIZATION

Juliano Cesar Alves Reis, Danilo Ciccone Miguel, Karen Caroline Minori Vieira

Universidade Estadual de Campinas - UNICAMP

American Cutaneous Leishmaniasis $(\mathrm{ACL})$ is a disease caused by protozoa of the genus Leishmania, transmitted by sandflies. ACL may, in its cutaneous form, present single or multiple lesions. Currently, its treatment includes pentavalent antimonials, amphotericin B and pentamidine. Although being a pathology of great epidemiological importance, its conventional pharmacotherapy does not always show good results and may cause a number of systemic side effects. In view of this problem, the present work, based on the need for more effective treatments, aims to explore the leishmanicidal potential of the plant Vernonia polyanthes Less. known as 'Assa-peixe'. This is widely used in folk medicine for antibiotic and antiinflammatory purposes. Recent studies have reported several bioactive substances in the extracts of 'Assa-peixe', suggesting their action against Leishmania. However, no effect on amastigote form has yet been described, which is the parasitic stage of clinical relevance in ACL. Therefore, this work aimed to establish the activity of these compounds against Leishmania (V.) braziliensis and Leishmania (L.) amazonensis. Hydroalcoholic extracts were obtained from the different organs of the plant, in addition to essential oil and alcoholic extract of the leaves. These compounds were applied at different concentrations in plates containing promastigotes of L. (L.) amazonensis for 24h. This experiment demonstrated important inhibitory activity of leaf essential oil (comp. 92) against promastigotes (about $60 \%$ reduction in viability compared to control at $100 \mu \mathrm{g} / \mathrm{mL}$ ). Hydroalcoholic leaf extract (comp. 84) also showed a relevant activity in the promastigotes being used as a parameter in subsequent experiments. The inhibition of viability by compounds 84 and 92 in L. (L.) amazonenis and L. (V.) braziliensis promastigote cultures during 24 48 and $72 \mathrm{~h}$ was then evaluated. There was no significant time-dependence relationship in reducing viability and compound 92 was more toxic to both species. The cytotoxicity of these compounds was also investigated in L929 fibroblasts and primary murine macrophages. These experiments showed higher cytotoxicity of compound 92 for promastigote forms when compared with fibroblasts and macrophages. In the current stage, in vitro infection assays are underway to evaluate the activity of the compounds against intracellular amastigotes and possible modulation in the microbicidal response of infected macrophages by the release of nitric oxide.

Keywords: American Cutaneous Leishmaniasis, Vernonia polyanthes Less. Bioactive substances.

\section{INFLUENCE OF ACUTE SECONDHAND SMOKE IN RENAL VASCULAR MORPHOLOGY}

Bárbara Valtudes Nogueira Thal, César Alexandre Fabrega de Carvalho

Faculdade de Medicina de Jundiaí

Smoking accounts for approximately 200,000 deaths per year in Brazil. With approximately 5000 toxic substances, the smoke that evolves from the burning tip of the cigarette is able to negatively influence nonsmokers who are in the contaminated environment, especially with nicotine. Among the affected organs are kidneys, which are highly vascularized and the maintenance of their functions depends directly on a good vascular permeability. Renal vascular changes are considered serious because they tend to cause internal homeostasis to rupture in a systemic way, besides being able to develop renal failure. Smokers, therefore, tend to have renal vascular lesions, but their relationship with secondhand smoke is not yet fully understood.

Keywords: Passive smoking; glomerulus; glomerular filtration; endothelium.

\section{TELEMEDICINE: CHALLENGES IN IMPLEMENTATION AND PERSPECTIVE FOR THE FUTURE}

Gabriela Moreira Gundim, Renata Calsaverini Leal, Ana Flavia Lacotis, Gabriel Henrique Cardoso Silva, Lais Amanda Matiazzi, Viviany Oliveira Simão

União da Faculdade Dos Grandes Lagos; São Leopoldo Mandic

Telemedicine refers to the use of telecommunications systems to provide distance healthcare. With the CFM resolution 1.643/2002 telemedicine became the medicine exercise with education, assistance and health research purposes, respecting CFM norms related to data transmission, confidentiality and guarantee of professional secrecy. In 2018, this resolution was revoked by the need of a more detailed analysis of all the critics and suggestions received. Therefore, the doctors procedures performed at distance was standardized, implementing technological and communication means that assures quality in the assistance provided to patients also including guarantees in data security, privacy and confidentiality and other premises required in telemedicine practice. This study brings a literature review of the challenges encountered in the implementation of the telemedicine in Brazil, also bringing perspectives of the future. For this research 226 articles published between 2002 and 2019 that met the criteria defined by the researchers, have been chosen. After the review, the analysis show that this innovation in the medical field has the potential to yield positive results on the health of the patients, also providing better access to healthcare and decreasing the cost of the services. However, at the same time the positive points are shown some negative effects stands out, like the substitution of the doctor-patient relationship and the trade of traditional medicine by other forms of technology. So as the number of apps and innovations in telemedicine keep growing, is important to understand the impact of these changes on the patients, healthcare professionals and future generations. By being a recent subject it is necessary to understand its impacts on the healthcare field and with this improve the procedures and care provided to the patients.

Keywords: telemedicine, implementation, challenges, future

Internal Medicine

17. HEMIMEGALENCEPHALY: CLINICAL PICTURE AND TREATMENT

Ricardo Ribeiro Homem Landi, Paulo Henrique Pires de Aguiar, Julia Magalhães Peixoto de Oliveira, Olivia Augusto Pereira Lima Pontifícia Universidade Católica de Campinas - PUC

Hemimegalencephaly is a brain congenital dysplastic malformation with abnormal growth of one of the cerebral hemispheres. The clinical presentation is variable, however, seizures and neuropsychomotor delay are common. The seizures are usually refractory to monotherapy drug treatment, which generates the need for a combination of multiple medications or surgical interventions, among which the most used are hemispherectomies. The goal of this study is to discuss the clinical condition of hemimegalencephaly and its treatments, by analysing the results and followup of reported cases. A systematic review was carried out to search for the keyword "Hemimegalencephaly" from 2002 to 2019 using the electronic platforms MEDLINE, PubMed, LILACS and Scielo, giving priority to review articles and case reports in English, Spanish and Portuguese. 44 
patients, 27 males and 17 females were analysed. Most of the cases were right hemimegalencephaly, of which $32,26 \%$ presented partial seizures, being the most frequent clinical manifestation in right HME cases. For right HME, most of the treated patients underwent surgical treatment with right functional hemispherectomy. For those patients, the surgical treatment was more effective in reducing seizure severity and frequency. Regarding the left HME cases, $46,16 \%$ presented epileptic muscle spasms as the main clinical manifestation. Most of these patients received drug treatment. There was an important reduction in seizure severity and frequency in only half of the cases. Analyzes showed that surgical treatment is more effective for seizure control than treatment with medication, especially when considering right HME. The follow-up after treatment is also very important as most reports showed that patients do not progress with complete remission of seizures or complete improvement of neuropsychomotor delay.

Keywords: Hemimegalencephaly, epilepsy, anticonvulsants, hemispherectomy

18. THERAPEUTIC REVIEW OF CUTANEOUS, KIDNEYS AND MUSCULOSKELETAL INVOLVEMENTS OF SYSTEMIC LUPUS ERYTHEMATOSUS

Patricia Sauchuk Ferreira, José Alexandre Mendonça, Letícia Novais dos Santos

Pontifícia Universidade Católica de Campinas

The Systemic Lupus Erythematosus (SLE) is an autoimmune and chronic disease characterized by the loss of immune self-tolerance involving the formation of autoantibodies and immune complexes resulting in inflammation of multiple organs and systems. The current therapeutic approach involves immunomodulation and immunosuppression, and also acts against specific organ manifestations. Despite the numerous advances in treatment, SLE patients still have a low quality of life, when compared with other patients diagnosed with other chronic diseases but yet, its premature mortality and morbidity rates continue to be high. The main aim of this literature review is to describe the current therapeutic strategies applied in the cutaneous, kidneys and musculoskeletal involvements of SLE. We ran a survey by using PubMed, Lilacs, and Medline and used "Lupus treatment" as keywords. Then, we selected 4 articles focused on the curren management strategies for SLE. The current treatment strategies, although of their relative effectiveness, are still limited by high failure and toxicity rates. Corticosteroids management therapy contributes to much of the longterm organ damage. Further studies on management strategies have to be reviewed, as an attempt, to reduce the undesirable effects described previously, pursuing the increase of long-term quality of life of patients diagnosed with this pathology.

Keywords: treatment, systemic lupus erythematosus, SLE.

\section{PREVALENCE OF OVERWEIGHT AND OBESITY IN DOWN SYNDROME}

Carolina Magalhães Britto Rodrigues, Priscila Donda, Gabriel Antônio Roberto, Ana Flávia Lacotis, Gabriel Henrique Cardoso Silva, Mariana Moraes Olímpio

União das Faculdades Dos Grandes Lagos

INTRODUCTION: Down syndrome is a genetic alteration that affects the development of the individual, as well as, it determines physical and cognitive characteristics. Most patients with the disease have the so-called simple trisomy 21. Several factors directly contribute to the increased risk of developing coronary heart disease such as acute myocardial infarction, hypertension, diabetes, lung disease, some cancers, kidney problems, gallbladder disease, joint, muscle and endocrine changes. Along with the increased life expectancy for individuals with Down syndrome in recent decades, obesity, respiratory disease (asthma, sleep apnea), musculoskeletal problems, liver disease, behavioral problems, social isolation, signs of depression, low self-esteem and Bullying is an aggravating risk factor for these individuals. OBJECTIVE: The present study aims to verify the prevalence of overweight and obesity, the correlation between the $Z$ and Percentile Drainage and Down syndrome of both sexes in children and adolescents, as well as in adults using the index. body mass (BMI). METHODOLOGY: A cohort study conducted during June 2017 with students enrolled in the Association of Parents and Friends of the Exceptional (APAE) in Mirassol, with a sample of 15 Down Syndrome patients, divided into two study groups, being the group one formed by children and adolescents and another group by adults. The anthropometric data collected were classified according to the BMI and Percentile graph, recommended by the Ministry of Health. RESULTS AND DISCUSSION: From the observed individuals, the adults were classified between 22 and 53 years old and children from 9 to 16 years old. There were 10 adults, $3(20.0 \%)$ females and 7 males $(46.7 \%)$. And 5 children, $2(13.3 \%)$ female and $3(20.0 \%)$ male. Only one adult $(10 \%)$ is classified as normal. Adults $5(50.0 \%)$ were classified as overweight, one adult $(10 \%)$ grade I obesity, two adults $(20 \%)$ grade II obesity and one adult (10\%) grade III obesity. One child $(10.0 \%)$ is classified as eutrophic. Already 5 children (90.0\%) were classified as overweight. No child was classified as obesity grade I, II and III. CONCLUSION It was observed that the degree of obesity in this total population between adults and children was $70 \%$ obese, $30 \%$ overweight and $20 \%$ normal, presenting a high rate of obesity or predisposition to this metabolic syndrome.

\section{CASE REPORT - TESTICULAR NHL RECURRENCE IN THE CNS OF ISCHEMIC HEART DISEASE PATIENT}

Bruna Calabrese Zanoni, Carla Adriane Roballo, Lara de Melo Ribeiro, Giulia Kodja Zanetta, Ana Raquel Okusu

Pontifícia Universidade Católica de Campinas - PUC

Patient A.T. , male, 60 years, with personal background of systemic arterial hypertension, coronary atherosclerosis with previous angioplasty in right coronary artery and non Hodgkin testicular lymphoma having it's curative chemotherapy treatment done in July 2018, following up at Puc Campinas' Hospital. In March 2019, returned to the service with holocranial headache associated with visual turbidity and lipothymia for two months. The investigation of the symptom was done performing a skull CT scan which has shown a massive tumor in the CNS, justifying the pacient's signs and symptoms of intracranial hypertension (ICH). Remaining under the care of the hospital's Neurosurgery team, it has been decided to collect a specimen of the tumor for biopsy and histopathological elucidation, so that the conduction of the case could be defined. During the hospitalization period, the patient evolved with typical precordialgia and joint follow-up was requested for Generalists' team. A new coronary angiography was performed showing restenosis of about $90 \%$ of the previous stent, with no possibility of intervention due to the patient's clinical performance at that moment.

Keywords: Non Hodgkin lymphoma; atherosclerosis; CNS relapse; palliative care; PET-CT.

\section{ACCUMULATION OF ADVANCED GLYCATION END PRODUCTS AND AGE ARE ASSOCIATED WITH THE PRESENCE OF DIABETIC DISTAL SYMMETRIC POLYNEUROPATHY}

Beatriz Martinelli Luchiari, Andrei Carvalho Sposito, Íkaro Soares Santos Breder, Vaneza Lira Waldow Wolf, Gabriela Machado

Pontifícia Universidade Católica de Campinas - PUC

INTRODUCTION: Diabetic distal symmetric polyneuropathy (DPN) is the leading cause of neuropathy in the world. Currently, there is an estimated prevalence of 422 million subjects with Type 2 Diabetes Mellitus (T2DM) worldwide and 7.6 million in Brazil, which approximately $50 \%$ show DPN after 10 years of disease. This comorbidity is a major cause of disability and amputation, although the only current preventive method is tight glycemic control. Given its importance, new risk factors should be investigated in order to address efforts to prevent the development of DPN. OBJECTIVES: To investigate the association between DPN and (1) accumulation of advanced glycation products (AGE) in tissues, (2) anthropometric measurements of body composition, mainly fat-free mass and android fat, (3) laboratory tests and (4) conventional risk factors including gender, advanced age, time of diabetes, high blood pressure, obesity, physical activity. METHODS: observational and cross-sectional analysis of 147 individuals aged 40 to 70 years with the diagnosis of T2DM, which are volunteers in a current Brazilian cohort. The presence or absence of PND was assessed by the Michigan Protocol, validated in Portuguese. The accumulation of advanced glycation end products (ACE) in tissues was assessed by measuring skin autofluorescence (SAF). Anthropometric measurements were evaluated using Dual Energy X-ray Absorptiometry (DXA). Laboratory tests were performed at the Fleury laboratory. Demographic, identification and lifestyle data, as well as weight and BMI, were collected at the study admission appointment. Statistical analyzes were performed using SPSS 20. Continuous data were compared by the MannWhitney $U$ and Pearson $X 2$ tests. The odds ratio by binary logistic regression and $p$ values less than 0.05 were considered statistically significant. RESULTS: Skin autofluorescence (OR: $0.430,95 \% \mathrm{Cl}: 0.228 ; 0.810, \mathrm{p}=0.009$ ) and age (OR: $0.931,95 \% \mathrm{Cl}: 0.883$; $0.980, p=0.007$ ) were significantly associated with the presence of PND, even after binary logistic regression. The presence of hypertension and obesity, gender, diabetes duration, physical activity, weight, BMI and laboratorial tests were not associated with PND in the study population. Fatfree mass, android fat and other anthropometric variables were not 
associated with PND. CONCLUSION: Accumulation of advanced glycation end products and age are associated with the presence of distal symmetric diabetic polyneuropathy in this cohort of Brazilian subjects with Type 2 Diabetes. Therefore, tight glycemic control with reduced glycemic variability plus low glycated hemoglobin level are important outcomes since diagnosis.

Keywords: Diabetic distal symmetric polyneuropathy, Type 2 Diabetes Mellitus, advanced glycation end products, skin autofluorescence, age.

22. STREPTOCOCCUS AGALACTIAE DISSEMINATED PYOMYOSITIS: A CASE REPORT

Mariela Guimaraes de Queiros, Djon Machado Lopes, Mario Jose Abdalla Saad, Cláudio Pillon Filho, Lenise Valler

Universidade Estadual de Campinas - UNICAMP

Pyomyositis is the primary infection of the skeletal muscles. It is more usual in adult men from the tropics, however there are cases reported in temperate zones. The clinical presentation is characterized by three clinical phases: invasive, purulent or suppurative and final stage, when most deaths related to this condition occur. Medical imaging such as computed tomography and magnetic resonance imaging are essential for diagnosis and clinical follow-up. The treatment consists of antibiotic therapy associated with surgical drainage of the muscular abscesses. The actual report describes the clinical evolution along 134 days follow-up of a 59 years-old male patient with diabetes mellitus who presented pyomyositis due to Streptococcus agalactiae group $A$ in the right sternocleidomastoid muscles, that projected to the thoracic cavity in addiction to minor abscesses in paravertebral lumbosacral and right femoral muscles.

Keywords: pyomyositis, acute myositis, pyogenic infection, Streptococcus agalactiae

23. ASSOCIATION BETWEEN SERUM VITAMIN D LEVELS AND ANTINUCLEAR ANTIBODIES IN PATIENTS FOLLOWED IN A CITY IN THE SOUTH OF MINAS GERAIS

Gabriel Aparecido Ferreira, Cristiane Mendes da Silva, Gláucia de Oliveira Moreira

Universidade Federal de Alfenas

Recent researches have addressed the relationship between vitamin $D$ levels and the function of the immune system. Studies have demonstrated that vitamin $\mathrm{D}$ may be related in the modulation of the adaptive and innate immune response. Studies performed in places where the population is under a low incidence of sunlight, the main mechanism by which the body produces vitamin $D$, have demonstrated a higher incidence of diseases involving the dysregulation of the immune system. Little is known, however, about the correlation between hypovitaminosis $D$ and the presence of autoantibodies, particularly the antinuclear antibody (ANA). The objective of this study was to evaluate the association between vitamin D levels and the presence of ANA in rheumatologic patients without a diagnostic of autoimmune disease, followed in an outpatient clinic in the south of Minas Gerais. The medical records of 400 patients were evaluated and the results of ANA obtained by Immunofluorescence in HEp-2 cells and the 25hydroxyvitamin D dosage performed by Electrochemiluminescence were collected. Patients were considered positive if ANA was reactive for dilution $>1 / 80$. Vitamin $D$ results were classified as deficient $30 \mathrm{ng} / \mathrm{mL}$. Tests were considered significant when $p<0.05$. Results: 65 patients were included in the study. The comparison of the general averages of vitamin $D$ between the FAN positive and negative groups did not present a significant difference $(p=0.406)$. The mean values of vitamin $D$ in the FAN positive group and negative showed a small difference in the deficient patients, but without statistical relevance $(p=0.112)$. Insufficient and normal patients showed no difference in mean vitamin $D,(p=0.656)$ and $(p=0.646)$ respectively. Conclusion: The association between vitamin D levels and the ANA result in the evaluated patients was not observed. New studies with larger sample sizes may reveal different results, opening up the opportunity for new studies on the relationship between vitamin $D$ and the presence of autoantibodies in the Brazilian population.

Keywords: Vitamin D; Autoimmunity; Antinuclear antibody

\section{OUTBREAK OF SCHIZOPHRENIA TRIGGERED BY CANNABIS USE}

Giovanna Uliana Rodrigues, Lúcio F. C. Ferraz, Bruna Lopes Ferreira de Souza Prado

Universidade São Francisco

INTRODUCTION: The consumption of cannabis as a psychoactive drug is alre ady widely known and practiced worldwide, much has been discussed about its legalization, in this context there is concern about its effects on health. In this article we will cover the relationship between the use of cannabis and outbreaks of schizophrenia among young people.0bjectives: the objective of this article is to investigate the possible causal factors and the relation of cannabis use and outbreaks of schizophrenia. METHODOLOGY: the methodology used was the bibliographic survey of articles from databases such as LILACS, PubMed, Scielo and Medline, published in the last 8 years in Portuguese and English. RESULTS AND DISCUSSION: among the findings is that the use of cannabis may exacerbate the symptoms of schizophrenia, and continuous use predicts the presence of more psychotic symptoms and worsening of the prognosis of those who already have schizophrenia. However, other factors must interact to increase the likelihood of developing psychotic symptoms, such as family history of schizophrenia, time of use, dose and age. However, studies also prove that the predisposition to schizophrenia does not predispose to greater use of cannabis. In youngsters abuse by the age of 18 provided a 2.4 -fold increased risk of developing psychosis late in life, moreover a study shows that exposure to synthetic cannabinoids in adolescent rats increased the susceptibility of schizophrenic hyperdopaminergic phenotype after puberty in relation to control rats. Conclusion: it is concluded that the use of cannabis is a risk factor, but not sufficient for the development of schizophrenia, other factors are fundamental, with genetics being one of the main factors. Therefore, there is still a need for more studies to prove the relationship between cannabis abuse among young people and the development of schizophrenia, since there are already indications of the causal relationship but it is not yet possible to alert the teennagers due to lack of a conclusive study.

Keywords: Schizophrenia; Cannabinoids; Cannabis; Marijuana Abuse.

25

\section{THE APPLICATION OF DIFFERENT KINDS OF KETOGENIC DIET IN THE} PREVENTION AND TREATMENT OF ALZHEIMER DISEASE

Enrico Crotti Pereira, Guilherme Chohfi De Miguel, Paola Fornazari Maniasso

Universidade São Francisco

The classic ketogenic diet (KD) is characterized by a high fat content, few carbohydrates and normal protein content and has some variations, as: Modified Ketogenic Diet, Modified Atkins Diet and more recently the Medium-Chain Triglyceride (MCT). The diet mimic the metabolic profile of fasting by reducing blood glucose concentration and increasing blood ketone bodies, like, $\beta$ hydroxybutyrate and acetoacetate, which will be the main energy source for the central nervous system. In the tradicional diet, the proportion of carbohydrates and fat is respectively, $55 \%$ and $30 \%$, while in the KD is $8 \%$ and $90 \%$. For almost 100 years, the KD has been used in the therapy nonfarmacologyc of epilepsy, however, recent studies have evaluated the KD application in other medicals conditions, like amyotrophic lateral sclerosis, traumatic brain injury, cerebral ischemia, cancer, diabetes and main neurodegenerative disorders, including Parkinson's and Alzheimer's disease (AD). Material and methods: Articles from the PubMed Scielo, MedLine and Google Scholar platform. Results and Discussion: analysis of benefits and harms of the different types of ketogenic diet. Conclusion: Classical presented the best option regarding risk-benefit, genetic component (Apoc), therapeutic and preventive coverage of Alzheimer's disease.

26. POLYCYTHEMIA RUBRA VERA: EPIDEMIOLOGIC ANALYSIS AND PATIENT FOLLOW UP ON A TERTIARY HOSPITAL OF CAMPINAS (SP)

Luiz Fernando Mohallem Reynaldo, Luciano Fuzzato Silva, Rodrigo Otero Coelho, Guilherme da Costa Boni Valente Pontifícia Universidade Católica de Campinas - PUC

Polycythemia Rubra Vera (PV) is the most common myeloproliferative disease, occurring in $1,9 / 100.000$ inhabitants (USA). The disease has a discrete predominance over men, grows in incidence after the 6th decade and occurs earlier in women. This retrospective and observational study was made with an analysis of 38 records from patients diagnosed with PRV. The data range from 1998 to 2019 and was collected using a protocol established by the researchers. The sample features $60,5 \%$ of male and 
medium age of 57 years. Observing the cardiovascular risk factors, the most prevalent was Arterial Hypertension (55,3\%). The most related clinical finding was Plethora $(68,4 \%)$. Five patients had thromboembolic events before the treatment and four occurred after it. As a treatment, almost all patients used hydroxyurea, with some using phlebotomy as prophylactic and/or maintenance option. Only four patients died during years studied. The disease manifests with symptoms occurring from blood hyperviscosity or vasomotor factors, such as fatigue, headache, visual disturbance and pruritus. Splenomegaly is the most common sign. Clinical findings can persist with laboratory control of the disease and can aggravate with its evolution. As such, clinical parameters are bad factors for patient's control. Given the unspecific clinical findings, the diagnosis is established following a set of criteria anchored in laboratory findings. The major criteria are high red blood cells count, suggestive bone marrow biopsy and JAK2 mutation. The minor criteria is reduced serum EPO, which diagnoses patients in cases of negative JAK2 mutation. Risk stratification is fundamental to establish the correct treatment, in an attempt of thrombosis prevention. The patient is considered as "high risk" if its age is over 60 years or it has a history of previous thrombosis. Modern protocols for "low risk" patients recommend phlebotomy until hematocrit target level is reached and daily low dose aspirin. For "high risk" patients the recommendation is hydroxyurea and to evaluate the response, preceded by phlebotomy until target hematocrit levels. Antiplatelets agents, anticoagulants, phlebotomy and other drugs can be used in special cases. The diseases' natural history is unknown mainly due to the established treatments that change its progression. Yet life expectancy is lower compared to the population. The patient's array showed epidemiology consistent with the literature. However, symptomatology data was shown to be lower. The phlebotomy usage happened in agreement with literature. Despite that, the usage of hydroxyurea in lower-risk patients disagrees with the protocols. None of the patients was considered intolerant or resistant to the therapies. The study showed a lower thrombosis rate than the literature, even though the treatment for low and high-risk patients lacked consistency with the literature.

Keywords: Polycythemia Rubra Vera; Symptomatology; Diagnosis; Risk Stratification; Treatment; Hematology.

\section{CASE REPORT: PSYCHIATRIC SYMPTOMS ON A PATIENT WITH} POLYCYTHEMIA VERA RUBRA

Guilherme da Costa Boni Valente, Luciano Fuzzato Silva, Rodrigo Otero Coelho, Luiz Fernando Mohallem Reynaldo

Pontifícia Universidade Católica de Campinas - PUC

Polycythemia Vera Rubra (PV) is a chronic monoclonal myeloproliferative neoplasia that affects the elder, increasing erythroid blood cells. It's global incidence is around $0.7-2.5$ in 10,000 people each year. The typical clinical manifestation consists in headaches, pletorism, dizziness, pruritus and splenomegaly, although the suspicion of the diagnosis is most frequent in asymptomatic patients after an altered blood test. Neuropsychiatric symptoms in PV patients are found in the literature, but not so prevalent in today's clinical practice. Old papers cite intense neurologic symptoms (hemiplegia) and psychiatric symptoms as characteristic of PV. Recent papers show fewer of these symptoms, which can mean a possible reduction of its prevalence. A male patient, 54 years old, went to the emergency room with symptoms of disorientation, bizarre attitudes, visual and auditory hallucinations, and persecutory delusion. Confronted by these initial symptoms, it was suspected of psychic delusion by a organic cause opting for stay in hospital to medicate and tests the patient. The patient was discharged after 3 days, in use of risperidone $2 \mathrm{mg}$ daily, coming back to the hospital after 4 days with the same symptoms and a new discovered family history of psychiatric problems of his brother. The patient was discharged after 6 days using risperidone $6 \mathrm{mg} /$ day and clonazepam $2 \mathrm{mg}$ a night. Kept an ambulatory follow up where he received treatment as if he was schizophrenic and showed less symptoms. After some time of follow up, he went the hospital again because of a paranoid disorder and was solicited news tests. It was opted for a phlebotomy on account of blood hyperviscosity. Then it was required a hematology consult, that saw the possibility of PV and stared a correct therapy. With the confirmed diagnoses and stated the correct therapy, the patient showed complete remission of the symptoms. Initial studies described the diseases as variable symptomatology with predominating neurologic symptoms. Nowadays, the clinical manifestations can be seen as neurological, because of symptoms like headache and dizziness, but findings like pletorism, pruritus and splenomegaly are seen a great amount, been considered classical symptoms of PV. It can be deduced that the popularization of hemograms, has allowed an early diagnosis, and a more effective treatment with hydroxyurea and aspirin have contributed for a downfall of the complicates cases PV. This case has been perceived as an atypical case, when compared with today's modern literature. The patient has shown only splenomegaly as a classical symptom, not been found headache, dizziness, pletorism and pruritus. The most prevalent symptom the patient presented was psychoses. Even though in the past more intense psychiatric and neurologic symptoms were shown, not only headache and dizziness. The case was in keeping with the literature. The only not consistent factor was the psychiatric family history, which is normally negative differing of this case in which it was positive.

Keywords: Polycythemia Vera Rubra; Psychoses; Case Report; Hematology.

\section{RETROSPECTIVE EXPLORATORY STUDY ON THE APPLICATION OF SPECTRAL DOPPLER IN SIÖGREN'S SYNDROME}

Caique Chagas Cavuto, José Alexandre Mendonça

Pontifícia Universidade Católica de Campinas - PUC

INTRODUCTION: when it comes to diagnosis and follow-up of patients with Sjogren's Syndrome (SS), the salivary gland ultrasonography (SCUS) has revealed relevant utility when compared to sialography and scintigraphy. Its specificity is also comparable to salivary gland biopsy. Doppler ultrasound is used to measure intravascular blood velocity and to differentiate structures from distinct echogenicity through grayscale (CS). Spectral Doppler (SD) is able to quantify inflammatory processes by calculating the internal resistance (IR) of vessels, since the inflammatory process predisposes a decrease in this IR. METHODS: analysis of previously recorded high-resolution SGUS images of 17 patients with SD and CS were evaluated to detect inflammatory changes of salivary glands. Objectives: the present study aims to correlate the sonographic findings with clinical and laboratory variables and evaluate the potential of diagnosis and complementary use of ED. RESULTS: A total of seventeen women with average age of $45.76 \pm 16.61$ years and disease duration of $4.35 \pm 3.21$ years. All patients presented xerostomia and xerophthalmia. The titration of antinuclear antibody (ANA) were $1 / 160$ to $1 / 1280$ with dense fine speckled nuclear pattern. of the patients studied, $88.24 \%$ had positive anti-Ro and titration above $211.76 \pm 79.70 \mathrm{U} / \mathrm{ml}$. Furthermore, $64.70 \%$ had positive anti-La and rheumatoid factor (RF) with titration above $111.11 \pm 140.71 \mathrm{U} / \mathrm{ml}$ and $127.17 \pm 308.56 \mathrm{IU} / \mathrm{ml}$, respectively. Erythrocyte sedimentation rate (ESR) value obtained was $29.82 \mathrm{~mm} \pm 10.81$ and $\mathrm{C}$-reactive protein (CPR) was $0.70 \pm 0.44 \mathrm{mg} / \mathrm{dl}$. With glandular scintigraphy, moderate to severe salivary excretion deficit was shown in $100 \%$ of the patients but $70.58 \%$ had positive gland biopsy. Spearman correlation: right parotid SCUS with ESR: $r=0.771$ and $p<0.00$; SGUS left submandibular gland with ESR: $r=0.551$ and $p=0.022$ SGUS right submandibular gland with ESR: $r=0.687$ and $p=0.002$; SGUS sublingual with FR: $r=0.585$ and $p=0.014$ : SCUS left submandibular gland with anti-La: $r=0.499$ and $p=0.041$; right parotid RI with anti-La: $r=0.642$ and $p=0.046$. CONCLUSION: USCS may be considered na importante tool in the evaluation of salivar glands in SS patients.

\section{Keywords: Sjögren's syndrome, Ultrasonography, Spectral Doppler}

\section{JUVENILE DIABETES- MANAGEMENT AND TREATMENT IN PUBLIC HEALTH NETWORKS OF ARARAQUARA-SP}

Beatriz De Giacomo, Mickaella Baldao Nechar, Maria Fernanda Maricondi Massari

Universidade de Araraquara

Its notable that a food plan, nutritional orientation and the practice of physical exercises are essencial for the treatment of diabetes mellitus, however, many challenges are faced in its adhesion, since most already have a defined lifestyle and the changes that must be made after the diagnoses will last for the rest of their lives. Besides that, family plays an essential supporting role, mainly when the diabetic is a child or an adolescent. The objective on this paper is to analyze the knowledge of diabetic adolescents that resides in the city of Araraquara and are treated in the public health system, the analysis was made using a questionnaire. The questions varied over the disease handling, ingested and restricted alimentation, medicines and the social impact of the disease. Observing the results, we identified that $14 \%$ of the children and adolescents felt uncomfortable about talking on their diabetes, evidencing how concerned they are about the disease and how it affects their social life. In what concerns the orientation about food comsumption, $87 \%$ of them feel well oriented, $93 \%$ knows the right meal times, and have family's support to follow a healthy diet, $80 \%$ consume fruits and vegetables and $74 \%$ follow a 
food plan according to what is recommended for a diabetic. Ultimately it was observed a contrast between the knowledge and concerns, what indicates us that although all of the necessary information about the treatment is available, they still feel fear and insecurities about the disease, this was exemplified by the high percentage when they were asked about the fear of feeling bad ( $53 \%$ ) and their affliction with the fact that they might be excluded from the social coexistance (20\%).

Keywords: Diabetes, child and juvenile, quality of life, feeding.

30.

\section{DISSEMINATED TUBERCULOSIS WITH ATYPICAL LYMPHNODE} INVOLVEMENT IN IMMUNOCOMPETENT PATIENT

Giovanna Thomé Streicher Souza, Isabella Siste de Almeida Aoki, Carla Adriane Roballo, Caroline Cardo, Manuela P. Cardoso

Pontifícia Universidade Católica de Campinas - PUC

INTRODUCTION: According to the WHO, Brazil is in 20th place in the world ranking of countries with the highest number of cases of tuberculosis (TB). Less than $2 \%$ of the population is affected by the widespread form of the disease. CASE REPORT: Female, 54-year-old patient with increased abdominal volume, nausea, vomiting, inappetence for 10 days, accompanied by weight loss. The patient presented jaundice and tachycardia (FC $113 \mathrm{bpm}$ ) and ascites. Abdominal computed tomography revealed enlargement of multiple lymphnodes and multiple hepatic and splenic nodules. Abdominal findings favored the diagnosis of infectious granulomatous disease. After one month, he returned for ultrasound-guided liver biopsy. The patient, during this period, reported significant abdominal discomfort and difficulty sleeping. The anatomopathological examination of the collected material of the hepatic biopsy showed portal fibrosis and formation of nodules, granulomas with gigantocytes in the portal space, coagulative necrosis in the focal area and mild central perivenulitis. The immunohistochemical study concluded positivity for histiocytic elements suggestive of mycobacteria. A therapeutic regimen was initiated for tuberculosis associated with corticotherapy. The treatment showed positive clinical and imaging response, emphasizing the reduction of lymph node enlargement and weight gain, without recurrence of ascitic fluid. DISCUSSION: Disseminated tuberculosis is defined as a finding of pulmonary lesion associated with one or more extrapulmonary foci, or more than two associated extrapulmonary foci. Clinical manifestations are nonspecific, including prolonged fever, ascites, abdominal pain, weight loss and cough. Predsponding conditions include alcoholism, HIV infection, malnutrition, advanced age, diabetes mellitus and immunosuppressive therapy. Mycobacterium tuberculosis has the lung as the gateway. In order for this form of tuberculosis (TB) to occur, the bacilli need to overcome the respiratory defenses, disseminating hematogenically to other organs. The differential diagnosis of this presentation includes infections (fungal, viral and bacterial), neoplasms and sarcoidosis. Extrapulmonary TB accounts for $10-20 \%$ of cases. Canglionic TB is the second most frequent manifestation, and the most usual form is the ganglionic involvement of cervical chains, being the bacilli able to reach the axillary ones. Cases of lesion in mediastinal, abdominal and diaphragmatic lymphnodes such as those of the reported patient are atypical. No tuberculin test was performed and no BAAR was found in the anatomopathological study of hepatic lesions. The treatment of choice is tuberculosis chemotherapy for six months, according to the Ministry of Health regulations. The report becomes pertinent due to the fact that disseminated TB to be responsible for less than $2 \%$ of the cases seen routinely in hospitals. This is an uncommon presentation because of the patient's age, the absence of a previous history of TB and HIV infection, and the lack of immunosuppressants. Conclusion: The present case clarifies the importance of including disseminated tuberculosis as a differential diagnosis. This form of clinical presentation of the disease is uncommon in immunocompetent patients, and a high level of suspicion is necessary to arrive at the diagnosis. The empirical treatment with tuberculostatics, despite the non-isolation of the causal agent, was positive, with clinical improvement and regression of the radiographic picture.

RETROSPECTIVE EXPLORATORY STUDY OF THE APPLICATION OF 3D, 2D ULTRASOUND AND HISTOGRAM IN ARTHROPATHIES

Isabella Siste de Almeida Aoki, José Alexandre Mendonça

Pontifícia Universidade Católica de Campinas - PUC

The use of ultrasound (US) in rheumatology has been growing, mainly because it is a low cost imaging method, without radiation exposure, and with good patient acceptance. Recently, a new modality has been studied to assist in the diagnosis of rheumatological diseases: three-dimensional (3D) ultrasound. It is a resource that still needs to be analyzed before its implementation, in fact, in clinical practice. The 2D ultrasound with high frequency linear probe, besides allowing a better resolution in the grayscale (SC) can characterize color image patterns, where it is possible to obtain a standard image histogram: "Indigo", "0range" or RGB, formed by additive primary color patterns, these being red, green and blue. Together with 3D US it can help to visualize detailed anatomical cuts, providing more accurate measurements of the studied lesion. Methods: A total of 16 patients $(37,5 \%$ males and $62,5 \%$ females), in which several osteoarticular lesions were detected. Objectives: The present study aims to detect echotextural damage through US 2D, 3D and histogram, correlating these measurements of area with volume through previously recorded images. Results: A total of 630 images was obtained, 470 were in $2 \mathrm{D}$ and 160 images in $3 \mathrm{D}$. In this study, all Spearman correlation coefficients were clinically significant $(p=0.002$ to $p<0.001$ ), with a strongly positive correlation between 2D US measurements in RGB, Indigo and SC color patterns. Conclusion: 3D and 2D US have great use in rheumatology and can better define ecotextural damage in various rhheumatologic diseases.

Keywords: Ultrasonography, inflammation, rheumatic diseases

\section{COMPARISON AND ANALYSIS OF MEDICAL STUDENT PERFORMANCE BETWEEN TWO ASSESSMENT METHODS USING DESCRIPTIVE STATISTICAL MODELS}

Matheus Santarosa Cassiano, Silvia Maria Riceto Romchim Passeri, Nelson Afonso Lutaif

Universidade Estadual de Campinas - UNICAMP

The need to build a homogeneous and objective evaluation process in the theoreticalpractical scope in disciplines involving clinical diagnostic skills is a constant challenge in the medical course. The discipline of Semiology and Propaedeutics, in its Internal Medicine internship, evaluated the student in theoretical exam, practical exam in the ward through case discussion, portfolio and concept evaluation. The difficulty of combining an assessment consistent with the content offered, the heterogeneity in the choice of patients for the practical test plus the problem of establishing objective criteria in the evaluation of this, portfolio and concept note established difficulties until 2016, due to the great loss. in the variability of the notes and the disparity with an expected Gaussian curve. Given this, a new format of practical evaluation was established, using actors who would simulate syndromes that should be diagnosed by students by anamnesis and physical examination of the device regarding the complaint. The calculation of the notes would be based on a standardized checklist. The project aimed to demonstrate that the evaluation consisting of a theoretical test and a practical test with simulated actors from the 2017 school year results in a normal distribution of the frequency of the practical test scores, as the standardization in the evaluation contributes to minimize the previous biases. of this test. While in 2016, the distribution did not follow normality; and in 2015 , the distribution was normal, but with low standard deviation, determining low grade variability and compromising the validity of the method. In addition, this study aimed to identify whether there is a correlation between the practical test and the student's academic performance coefficient over the course of graduation from the Person correlation coefficient for normal distributions and Spearman's for nonnormal distributions. The result was weak, but significant in 2016, and showed no correlation in 2015 and 2017, demonstrating the bias of the yield coefficient, as it is a note composed of evaluations with little or no consolidated standardization degrees.

Keywords: Medical education; semiology and propaedeutics; evaluation; internal medicine.

\section{PROFILE OF STROKE PATIENTS IN A PHILANTHROPIC HOSPITAL IN ARARAQUARA - SP.}

Rafaela Abreu dos Santos, Júlia Jodjahn Figueiredo

Universidade de Araraquara

Since the last decades, two processes have been observed in Brazil: the demographic transition and the epidemiological transition leading to the increase of noncommunicable chronic diseases, such as Stroke. These processes have determined important changes in the profile of diseases that affect the population. Health professionals need to be prepared and organized for the fast and safe care of these patients, seeking to prevent complications and sequelae. People should also be able to recognize early signs and symptoms of stroke and the need for immediate help. We performed a prospective analysis of 30 medical records from April 2018 to January 2019. An association of $84 \%$ was found between patients who had 
ischemic or hemorrhagic stroke and who had Systemic Arterial Hypertension An important association was found between smoking (46\%) and Diabetes Mellitus ( $46 \%$ ) and the occurrence of stroke (ischemic or hemorrhagic). The most prevalent signs and symptoms were also associated in the results, with Mental Confusion (61\%) being the most reported sign and symptom in the medical records analyzed. In addition, socio-demographic factors such as patient origin and marital status were analyzed, with $47 \%$ of married patients. It was possible to conclude from the analyzed data that, as expected, there is a high association between risk factors and stroke in the patients studied, especially with hypertension, diabetes mellitus and smoking.

Keywords: Cerebrovascular accident; Epidemiological transition; Hypertension

\section{Gyneco-Obstetrics}

34. MAGNESIUM SULPHATE IN PREECLAMPSIA WITH SEVERE FEATURES: EVALUATING ADEQUATE USE, MATERNAL AND PERINATAL OUTCOMES Amaro, L. B., Harfuch, B. R., Guida, J. P., Costa, M. L.

OBJECTIVES: To describe the maternal and perinatal outcomes in cases of preeclampsia ( $\mathrm{PE}$ ) with severe features treated with magnesium sulphate, and to describe characteristics of the medication use (triggering factor, average time of use, doses, adverse effects and need of new administration). METHODS: Descriptive study, with selection of all pregnant or postpartum women that received magnesium sulphate for treatment of PE or Eclampsia at the Women's Hospital (CAISM/Unicamp) over a one yea period (2017). Cases of magnesium sulphate were selected from the computer based medical records of prescriptions and further selected if PE. The medical records related to these cases were extensively reviewed fo data collection. All data (sociodemographic resources, clinical and obstetric history, diagnosis of PE and diagnosis of severe features, maternal and perinatal outcomes and conditions of use of magnesium sulphate) were entered into the study database and analyzed using Excel for Windows. Continuous variables were used as average and standard deviation, and categorical variables were included in percentage (\%) of frequency. RESULTS During the study period (2017), 171 cases of PE with signs of severity were included. The average age was 28.73 years and almost half $(49.7 \%$ ) had comorbidities, of which $57 \%$ were chronic hypertention. At the diagnosis of preeclampsia, patients had an average blood pressure of $165 / 102 \mathrm{mmHg}$ and $70.9 \%$ presented significant proteinuria $(>0.3 \mathrm{~g} / 24 \mathrm{~h})$. Most women presented severe symptoms $(64.3 \%)$ or severe hypertension $(61.4 \%)$. The frequency of eclampsia and HELLP syndrome among the cases was $8.2 \%$ and $14 \%$, respectively. In most cases (77.8\%), magnesium sulfate use started before labor (average gestational age of 33.75 weeks), 9.9\% presented a mild adverse effect $(7.5 \%$ had oliguria, $3.5 \%$ absence of tendinous reflex, $1.2 \%$ nausea and $0.6 \%$ vomiting). Among the cases, $55.2 \%$ evolved to preterm labor, $83.3 \%$ were cesarean and the average gestational age of delivery was 35.13 weeks. Only $3.9 \%$ of the newborns had an Apgar at 5 minutes score below $7,17.6 \%$ required hospitalization in neonatal ICU and $75.8 \%$ did no describe neonatal complications. However, there were 5 neonatal death and 5 stillbirths among the considered cases. CONCLUSION: Magnesium sulfate use was not associated with serious adverse effects. Its use in cases of early PE can help to ensure clinical safety and proper management in deciding the best time for childbirth, reducing frequency of premature birth (specially early preterm and extreme preterm births), and severe materna complications.

Key-words: preeclampsia, eclampsia, treatment, magnesium sulfate

PREECLAMPSIA IN BRAZIL: SYSTEMATIC REVIEW AND META-ANALYSIS

Luis Gabriel Pissinatti, Caio Augusto Hartman, José Paulo de Siqueir Guida, Beatriz Gadioli Andrade, Bruna Fagundes Rodrigues, José Paulo de Siqueira Guida, Caio Augusto Hartman Faculdade de Medicina São Leopoldo Mandic

INTRODUCTION: Preeclampsia is the main cause of maternal morbidity and mortality and an important cause of prematurity in Brazil. However, there is no national surveillance network to assess its occurrence. OBJECTIVE. To evaluate the incidence of preeclampsia and its main complications (eclampsia, Hellp syndrome, maternal death, perinatal death and prematurity) in Brazil. Methods: Systematic literature review in 3 different databases (PubMed, Scielo and Embase), until July 2019, without language restriction, using the following terms: preeclampsia, eclampsia, hellp syndrome, hypertension in pregnancy, hypertensive disease of pregnancy, hypertension in pregnancy. Selected longitudinal studies that reported at least the frequency of preeclampsia. The studies were included in RevMan 5.0 and their results were pooled. Ethical evaluation was waived because it deals with analysis of studies already published. RESULTS: 237 studies were identified, 27 selected after reading the abstracts and 5 included after the evaluation regarding the inclusion criteria, totaling 11639 women evaluated The frequency of preeclampsia was $400 / 11639$ (3.42\%); 2 reported the frequency of eclampsia $(0.15 \% ; 8 / 5496) ; 2$ perinatal death $(0.45 \% ; 8 / 1769)$. 2 of prematurity $(1.3 \% ; 23 / 1769)$. Other outcomes were not reported. CONCLUSION: The frequency of preeclampsia and prematurity, but not that of perinatal death, was below the world average. Standardization of results in preeclampsia studies is critical for grouping results.

Keywords: Preeclampsia. Eclampsia. Hellp syndrome. Maternal death. Perinatal death. Incidence. Brazil.

\section{HORMONAL CONTRACEPTIVE METHOD: THE REASONS FOR A DAMAGED REPUTATION}

Carolina Fernanda Sgobetta, Fernanda César Rennó Coelho, Carlos Alberto Maganha

Faculdade de Ciências Médicas de São José dos Campos - Humanitas

The contraceptive is a method that aim to prevent an unwanted pregnancy, however, its side effects are expressive in the woman's health, which causes the generation $Y$, "millennium generation"; it is failing to use it. Thus, the purpose of this article is to understand the reasons why the modern woman is failing to adhere to hormonal therapy. It is a study of bibliographic review using books and scientific articles published in databases of biomedical bibliography, in addition, the questionnaire present in the article will be used for future field research. According to the literature, the harms of oral contraceptive use were verified, supervising its benefits. Thus, it is concluded that this drug is harmful to female health.

Keywords: oral contraception, $Y$ generation and adverse effects.

\section{7. \\ PRIMARY VAGINAL SQUAMOUS CELL CARCINOMA: CLASSIC PRESENTATION OF A RARE TUMOR}

Mariana Castro Locateli, Luíza Paganelli, Laisa de Aguiar Costa Cesar, Tauane de Lima Fiorillo, Eduardo Venerando da Silva Universidade de Araraquara - UNIARA

OBJECTIVE: To report the case of a patient with primary vaginal carcinoma. CASE REPORT: Female patient, 43 years old, referred to Santa Casa de Araraquara in May 2018 due to vaginal bleeding associated with a fetid odor 3 months ago. Reports weight loss of 4 pounds in the last 6 months. On physical examination, a fragile lesion in the posterior vagina was observed. A biopsy revealed welldifferentiated invasive squamous cell carcinoma. After failure of the initial treatment and worse progression of the condition and after the results of the exams and the general condition was given after an approach with palliative care team with priority in the control of symptoms, wich follow her up until June 18th when was declared hers death. Conclusion: It was concluded that vagina carcinoma is a rare entity, representing 1 to $2 \%$ of gynecological malignant tumors, being necessary for its diagnosis the exclusion of primitive neoplasms of other organs, specifically cervix, vulva, endometrium, colorectal and bladder.

\section{Keywords: vagina, primary, carcinoma}

\section{BALLANTYNE SYNDROME IN THE SECOND TRIMESTER OF GESTATION} Pedro Nogarotto Cembraneli, Julia Brasileiro de Faria Cavalcante Faculdade de Medicina FACERES

INTRODUCTION: Ballantyne syndrome (BS) is characterized by fetal hydrops, placentomegaly and maternal edema. It is a rare syndrome, occurs between the second and third trimester of pregnancy and has a high morbidity and mortality. CASE REPORT: Pregnant woman, 20 years old, 26 weeks of gestation, had lower limb edema. On examination, she presented stable vital signs, uterine height of $28 \mathrm{~cm}$, fetal heartbeat $130 \mathrm{bpm}$. Ultrasound showed severe fetal hydrops with pleural effusion, fetal ascites, placentomegaly, pathological A-wave venous duct. $63 \mathrm{~cm} / \mathrm{sec}$ mean brain Doppler. Laboratory tests showed hemoglobin of 10.1 and numerous bacteria in the urine. We opted for hospitalization and treatment of the infection. During hospitalization, the patient evolved with preeclampsia (150x100) and absence of the fetal heartbeat. With the confirmation of fetal death, induction was performed vaginally and later curettage of the uterine 
cavity. Patient evolved well after surgery, with complete regression of edema. DISCUSSION: BS has its unknown pathogenesis and uncertain pathophysiology. Clinical manifestations are variable, but usually patients have edema, rapid weight gain, progressive dyspnea and hypertension, a similar presentation and clinical course for preeclampsia. However, in contrast to preeclampsia, dilutional anemia is common (preeclampsia is usually hemoconcentration). CONCLUSION: Fetal hydrops is associated with a higher risk of preeclampsia. The definitive treatment is delivery regardless of gestational age, due to the high morbidity and mortality of the disease.

Keywords: Ballantyne Syndrome; Placentomegaly; Fetal hydrops.

39.

CERVICAL CARCINOOSARCOMA - THE DIFFICULTY OF ESTABLISHING THE DIAGNOSIS: CASE REPORT

Cesar Caue Bassetto, Lucas Pivetta Genovez, Joao Pedro Branco Santana, Gabriel Lucas Martins, Carolina Fernanda da Silva

Cervical carcinosarcoma is a rare neoplasm with approximately 62 cases reported in the English literature. The disease in question is quite aggressive, composed by both tissues of epithelial and mesenchymal origin, and the sarcomatous component is derived from the malignant metaplastic epithelium. It affects women more advanced, between the sixth and seventh decades of life, and mainly black. Symptoms include abnormal vaginal bleeding, pelvic pain, and enlarged uterus. The diagnosis is difficult to perform, requiring a correlation between biopsy findings and immunohistochemical examination. This is a report of a 58-year-old white patient with uterine cervix carcinosarcoma diagnosed in stage IIb, presenting as an initial symptom abnormal vaginal bleeding. HPV and Chlaymidia trachomatis were both negative, using the hybrid capture technique. The diagnosis was established after hysteroscopy biopsy and immunohistochemical examination.

\section{Pediatrics}

40.

TRACHEAL BRONCHUS - PULMONARY CONGENITAL ANOMALY IN PATIENTS WITH DOWN SYNDROME

Beatriz Sosa Pires da Silva, Patrícia Sauchuk Ferreira, Maria de Fátima Campos Marciano

Pontifícia Universidade Católica de Campinas

Tracheal bronchus is an anatomical malformation common in patients with Down Syndrome, usually asymptomatic and an incidental finding in bronchoscopies. This case report is about a patient with Down Syndrome and recurrent pneumonia, who underwent several thorax radiographies which revealed persistent right upper lobe atelectasis. A tracheal bronchus was diagnosed after bronchoscopy. It was decided that this patient should be treated in a conservative way, with respiratory physiotherapy and inhalatory mucolytics, which have improved the patient's condition. It is known that children with Down Syndrome are commonly hypersecretive and have deficient immunologic response, which is why they need more assertive conducts to have better life quality.

Keywords: tracheal bronchus, Down Syndrome, conduct.

41.

ABDOMINAL PAIN IN CHILDHOOD: EVALUATION OF CASES REFERRED TO TERTIARY HOSPITAL

Gabriela Parússolo Martins, Maria Ângela Bellomo Brandão

Universidade Estadual de Campinas - UNICAMP

A complaint of chronic abdominal pain has a very variable presentation and complex etiology, and may be of organic or functional cause. Thus, the lack of understanding of the physiopathology and proper treatment still leads to extensive investigations, therapeutic treatments, patient dissatisfaction and health care costs, and when there is no resolution in the primary and secondary levels of health care, cases are referred to the tertiary level hospital. In this context, the present study - which has a retrospective observational character with cross-sectional, qualitative and quantitative analyzed the data recorded from 2013 to 2018. The sample consisted of cases of chronic abdominal pain registered at the Hospital de Clínicas of the State University of Campinas. Among the results obtained from 328 participants, there was a prevalence of females, median age of 9 years, patients were nourished (even with abdominal pain with a median duration of 1 year) and $70 \%$ were diagnosed as having functional etiology (including functional abdominal pain, functional dyspepsia, abdominal migraine and constipation), data compatible with the high prevalence of functional diseases in this age group and which, when diagnosed, can be conducted in primary care services. Thus, a better understanding of chronic abdominal pain and referrals within the care network can contribute to the proper management regarding referrals, diagnoses and treatments of children with chronic abdominal pain.

Keywords: children, chronic abdominal pain, Rome IV.

\section{SYSTEMATIC INVESTIGATION OF INTESTINAL HABIT OF CHILDREN WITH CYSTIC FIBROSIS}

Diane Chou, Elizete Aparecida Lomazi

Universidade Estadual de Campinas - UNICAMP

Changes in $\mathrm{HI}$ are considered in the etiology of gastrointestinal symptoms that negatively affect the life quality of Cystic Fibrosis patients. BACKGROUND: Characterize the HI from preschooler's children that were diagnosed with Cystic Fibrosis. METHODS: Systematic Inquiry about the evacuations frequency, stool consistency and appearance. The appearance was rated subjectively and by Bristol pediatric visual scale, that featured the stool in five aspects: 1. Cymbals, 2 . Shape of a lumpy banana with cracks, 3. Shape of a soft and smooth banana, 4. Soft, but without a shape, 5. Diarrheal. The patients elected were up to seven years, being treated in outpatient follow-up at a specialized center in Cystic Fibrosis, where are around ninety children assisted, identified by neonatal screening with confirmed diagnosis (sodium dosage / chlorine in sweat and genetic study. The pancreatic function was assessed by fecal elastase dosage (ref.: > 100 $\mu \mathrm{g} / \mathrm{g}$ stool). Ostomy patients were excluded from the survey. Also were excluded the ones that was not possible to fill the form completely. RESULTS: Caregivers of 49 children answer the form, being 25 girls, average age of 3,5 years (min 0.09 and max. 7.6 years). Most of patients (46/49) presented pancreatic insufficiency and were using pancreatic enzyme, in doses between 5 and 10 thousand lipase units / $\mathrm{kg} /$ day. The frequency of bowel movements was from 1 to 4 times a day. 16/49 patients mentioned daily frequency. Stool consistency was referred to as pasty in $24 / 49$ patients or parched with cracks, or also in cymbals on 25/49. The Consistency assessment by visual scale was consistent with the subjective information, to know: Bristol 1 or 2: 25 patients, Bristol 3 or $4: 21$ patients and 5 on 3 patients. On the three patients with pancreatic sufficiency, the consistence of the stool was described as hard and the aspect classified as Bristol 1. CONCLUSION: About half of the assessed children were identified changes in stool appearance and consistency, despites de daily frequency been one or more times a day. Early attention to this condition may prevent progression to constipation and fecal impaction on the patients affected by Cystic Fibrosis

\section{PEDIATRIC FLEXIBLE VALGUS FLATFOOT CORRECTION BY ARTHROEREISIS}

Maria Júlia Palitot de Melo, Bruna Madeleine da Silva Simplício Flôr, Alexandra Mauriel dos Reis, Débora Penna Chaves Bertazzo, Maria Fernanda Rosa Dezan, Cíntia Kelly Bittar Pontifícia Universidade Católica de Campinas

OBJECTIVE: This article aimed to evaluate the efficacy of arthroereisis with the use of synthetic polyethylene implants as a method to correct excessive valgus deformity in pediatric patients with flexible valgus flatfoot. METHOD: Retrospective study of 20 pediatric patients with flexible valgus flatfoot, being 23 feet (three bilateral cases), from five to 14 years old, operated between January 2009 and July 2016. Clinical evaluations were performed on the basis of the Valenti podoscopic classification and the American Orthopaedic Foot a Ankle Society criteria, based on radiographic images and podoscopic analysis. These patients underwent surgical treatment with the introduction of a synthetic implant (polyethylene conical screw) in the sinus tarsi. Multiple linear regression analysis with Backward selection of variables, the Bordelon, Kite, Gould, Meary and Pitch angles of the pre and postoperative of radiographic images of the patients submitted to arthroereisis were performed. RESULTS: The arthroereisis with interposition of synthetic material was satisfactory, considering that 21 of the feet $(91 \%$ of the cases) presented clinical and radiographic improvement, with correction of angles and improvement in deformity degrees. Two cases presented implant loosening. The variables of the Bordelon and Pitch angles significantly influenced $(p<0.05)$ the improvement of the correction of deformity degrees. CONCLUSION: The arthroereisis with the interposition of synthetic polyethylene material in the sinus tarsi showed to be a minimally invasive and effective technique for flexible flatfoot in symptomatic pediatric patients.

Keywords: Synthetic implant, Flatfoot, Arthroereisis 
44. THE IMPACT OF NUTRITIONAL STATUS ON PERIOPERATIVE OUTCOMES IN INFANTS WITH OR WITHOUT DOWN SYNDROME SUBMITTED TO CARDIAC SURGERY.

Letícia Muniz de Vargas, Luiz Fernando Caneo, Fernando Antoniali, Aída Luiza Ribeiro Turquetto, Letícia Teixeira Marcondes, Aída Luiza Ribeiro Turquetto, Fernando Antoniali

Pontifícia Universidade Católica de Campinas

BACKGROUND: Malnutrition is common in infants with congenital heart disease (CHD). Children with CHD are considered high nutritional risk due to clinical conditions inherent the disease. OBJECTIVE: To analyze the association of nutritional status and morbidity and mortality in infants with or without Down syndrome submitted to corrective heart surgery. METHODS Retrospective study involving 1,116 patients reported to the ASSIST Registry among 2014 to 2018. For classification of nutritional status was used Z-score index curves, from World Health Organization, of Body Mass Index (BMI) for Non-Syndromic Infants (NSI) was used specific weight curves of Bertapell et al for infants with Down syndrome (IDS). NSI were considered malnourished when they were below Z-score -2 BMI and IDS were considered malnourished when they were below the 3 rd percentile and, in nutritional risk, between the 3 rd and 10th percentiles in the Down syndrome curve. RESULTS: There was no connection between nutritional status and mortality in either group. At the NSI group, $33.6 \%$ were malnourished, and $47.7 \%$ were diagnosed with septal defect (SD); $7.5 \%$ had pulmonary hypertension (PH) and $46.6 \%$ were admitted into an intensive care unit (ICU) preoperatively. The malnourished NSI had more extubation failure (OD = $3.26, \mathrm{p}=0.040$ and $\mathrm{Cl}=1.05-10.0)$; longer mechanical ventilation $(\mathrm{MV})(\mathrm{p}=$ 0.002 ) and longer hospitalization in the ICU $(p=0.003)$. In the IDS group $35.7 \%$ were malnourished or at nutritional risk, $40.4 \%$ had the diagnosis of SD, $15 \%$ had PH and longer hospitalization ICU after surgery $(p=0.035)$. Conclusion: Although malnutriton did not impact in hospital mortality, was found a higher frequency of extubation failure, longer MV and longer time admitted in ICU.

Keywords: Malnutrition, infants, congenital heart desease, surgery and Down syndrome.

\section{MALNUTRITION MORTALITY PROFILE IN THE PEDIATRIC POPULATION OF} BRAZIL

Luísa de Melo Brandão, Samara Nidale Karaja, Maria Carolina Seixas, Leticia de Carvalho Brito, Julia Nappo, Lucélia Martins Pinto Melgares UNICEUB

INTRODUCTION: The pediatric malnutrition is, according to the WHO, one of the most common causes of pediatric mortality and morbidity in Brazi reaching rates as high as $20 \%$ of all deaths at hospital levels. OBJECTIVE: This study was developed to evaluate how, over 10 years, mortality by malnutrition in the pediatric group affected the quality and durability of brazilians from the age of 0 to 19 years. METHODS: It is a descriptive time series study based on data from the Departamento de Informática do Sistema Único de Saúde (DATASUS). Data was collected regarding mortality from malnutrition in the pediatric population of Brazil, of both boys and girls, between the ages of 0 and 19 years old, from May 2009 to May 2019 DISCUSSION: Malnutrition can be classified as a clinical condition due to relative or absolute deficiency or excess of essencial nutrients. Thus, it can be subdivided into primary, with quantitative or qualitatively insufficient nutrition in calories and nutrients, or secondary, by changes in energy needs or other factors not directly related to the diet, accompanied by insufficient intake. Most cases occur in kids under the age of 5 , which may compromise their psychological or physical development. Results: On the observed data, it was noticed that there was an initial increase followed by a small reduction in the proportion of deaths from malnutrition, with 91 deaths reportes in 2009, followed by a rise to 166 deaths in 2010 and a subsequent fall to 111 in 2019. Most cases of death occurred in the Northeast region. From the data obtained from the SHI/SUS, it has been found that, during the analyzed period, 1305 deaths from malnutrition occurred in Brazil. The age group of under 1 year old was the most affected, with 766 deaths $(3,11 \%$ of the total). In 2009, 94 deaths were notified, a number that got considerably higher in 2010 and fell again in 2018, reaching 111 deaths. The highest absolute number of deaths occurred, as discussed before, in the Northeast region, where numbers as high as 545 deaths, which represents $2,43 \%$ of the total, were notified. The national mortality rate was $2,10 \%$, being the highest proportion registered in the North region $(2,46 \%)$ and the lowest in the South region $(1,13 \%)$.

\section{FAHR'S SYNDROME IN CHILDHOOD: A CASE REPORT}

Estela Macias Castilhos, Maria Laura Thomaz Rossi Romani, Bruna Durigan Sirian FACERES

Fahr's syndrome, a degenerative neurological disease, has the most common age group between 40 and 50 years, but can occur in childhood Calcium deposition in the cerebral parenchyma is essential for the diagnosis, besides laboratory alterations of this electrolyte. It may be asymptomatic or present symptoms such as seizures and tetany. The report deals with an eight-year-old child with a history of seizures, presenting subcortical calcifications in base nuclei and white matter on brain MRI, changes in calcium metabolism, and delayed neurodevelopment.

\section{CARDIOVASCULAR ALTERATIONS IN TURNER SYNDROME AND KARYOTYPE- PHENOTYPE CORRELATIONS}

Marcela Bruni Ratto, Sofia H. V. Lemos-Marini, Renata Isa Santoro, Andréa Trevas Maciel Guerra

Universidade Estadual de Campinas - UNICAMP

Turner syndrome (TS) is characterized by the presence of an $X$ chromosome and total or partial absence of the second sex chromosome ( $X$ or $Y$ ) and leads to short stature, primary hypogonadism, various dysmorphisms and malformations. Congenital and acquired cardiovascular diseases are found in about $20 \%$ to $30 \%$ of cases and are the leading cause of death. They are usually related to the left side of the heart (aortic alterations such as bicuspid valve and coarctation, and mitral disorders) and can lead to lifethreatening complications such as infectious endocarditis, valve dysfunction, aortic aneurysm, and thoracic aortic rupture. The incidence of congenital cardiovascular diseases is higher in patients with the $45, \mathrm{X}$ karyotype $(39 \%)$ than in those with mosaicism or structural abnormalities of the sex chromosome $(X)$, such as isochromosome $X q$ (11-12\%). However, little has been elucidated about the cause of such disparity, and there are few studies correlating the findings with other karyotypes found in this syndrome. The aim of this study was to broaden the knowledge about these anomalies in TS, determining their frequency and establishing correlations between karyotype and phenotype. One hundred patients with TS confirmed by karyotype who were attended at the Hospital das Clínicas, State University of Campinas, were selected. Data were obtained on echocardiogram findings from the last visit to the service or, in patients who had cardiac surgery, from the last examination performed before surgery. In total, our sample was composed by 80 patients who had an echocardiogram performed. There were structural cardiac alterations in 27 of the 80 cases $(33.8 \%$ ), with predominance of coarctation of the aorta and bicuspid aortic valve, and the presence of these anomalies was associated with signs of jugular lymphatic obstruction sequence. The $45, \mathrm{X}$ karyotype was associated with aortic anomalies, but not with anomalies of mitral valve. In the group of patients without structural changes, there were no differences between $45, \mathrm{X}$ and other karyotypes regarding ejection fraction, systolic shortening fraction and left ventricle (LV) mass index. When compared to general population, ejection fraction was reduced in $56 \%$ of cases, LV mass index was reduced in $30 \%$ and increased in $15 \%$, and aortic diameter was reduced in $7 \%$ and increased in $21 \%$ of the cases. Our findings regarding structural cardiac alterations and their predominance in karyotype $45, \mathrm{X}$ are similar to those in the literature, but the wide karyotype heterogeneity makes it difficult to get more specific karyotype-phenotype correlations, which depend on a larger sample. Changes in LV parameters and aortic diameter in a significant portion of cases also indicate the need for careful cardiac follow-up even in patients without structural abnormalities.

Keywords: Turner syndrome, congenital heart diseases, bicuspid aortic valve, aortic coarctation, echocardiography

48. ASSOCIATION BETWEEN THE BACTERIA STREPTOCOCCUS PYOGENES AND THE OCCURRENCE OF RHEUMATIC FEVER

Mariana Cabriela Apolinario Mian, Ana Beatriz Franco Arena, Vinicius Roberto Martins Bragadioli, Roger Teixeira Rodrigues, Jeferson Hohne Debora Giacomini

EDUCLAR - Centro Universitário Claretiano

INTRODUCTION AND OBJECTIVE: This work aimed to address the association between the bacteria Streptococcus pyogenes and its most serious development, rheumatic fever. METHODS: The bibliographic survey was carried out from May to June of 2019, in the databases: Medical bibliography (MEDLINE), Online Electronic Scientific Library (Scielo). It was also consulted 
the databases of the Ministry of Health and the Brazilian Society of Pediatrics and Brazilian Society of Cardiology. The keywords "Streptococcus pyogenes", "rheumatic fever" and the corresponding English term "Rheumatic fever" were used. After reading, we excluded those that were not related to the purpose of this study, resulting in the selection of 14 articles. DISCUSSION: Rheumatic fever resulting from $S$. pyogenes proliferation is considered a sequel and can lead to myocardial and heart valve damage. Its occurrence is of an autoimmune nature and its episodes occur at intervals of 1 to 3 weeks after having pharyngotonsillitis coming from S. pyogenes bacteria in genetically susceptible and relapsing hosts. It is characterized by non-suppurative inflammatory lesions involving the cardiac muscle tissue, the joints, the subcutaneous cellular tissue and the central nervous system. Studies of the prevalence of rheumatic fever demonstrate the predisposition of patients who presented an episode of rheumatic fever to new episodes as a consequence of subsequent streptococcal infections of the respiratory tract. In the literature review of the pathogenesis of the disease, the most accepted assumption is the existence of antigens common to cardiac muscle tissues and to certain streptococcal cell structures such as $M$ protein of the cytoplasmic membrane. In developing countries, it presents high rates of morbidity and mortality among individuals aged 5 to 15 years. In Brazil, the prevalence of rheumatic fever is 3 to $5 \%$ among children and adolescents. The low socioeconomic power, poor access to health services and malnutrition are factors that corroborate for these rates. RESULTS: The most affected audiences are children and adolescents. The symptoms reported in the objects of study are carditis, arthritis, chorea, subcutaneous nodules, arthralgia and fever. It is concluded that the greatest sequel from a previous infection caused by the streptococcus bacteria is rheumatic fever and, therefore, the article addresses the association of the same. The treatment was investigated. Conclusion: It was observed the ineffectiveness of the Unified Health System (SUS) to quantify the incidence and prevalence of bacterial pharyngotonsillitis caused by streptococcus. Further studies involving the Streptococcus pyogenes bacteria and the occurrence of rheumatic fever should be encouraged.

Keywords: Streptococcus pyogenes, rheumatic fever, streptococcal pharyngotonsillitis

49. NEUTROPENIA IN CHILDHOOD - CLINICAL AND EPIDEMIOLOGICAL CHARACTERIZATION OF PATIENTS TREATED AT A TERTIARY CENTER Fernanda Rodrigues Molles, Adriana Gut Lopes Riccetto Universidade Estadual de Campinas - UNICAMP

Neutropenia is defined as a decrease in the number of neutrophils in the bloodstream. They are relatively common in the pediatric age group; usually associated with recurrent infections, their investigation is often flawed or nonexistent. The characterization of the clinical and epidemiological aspects of pediatric patients diagnosed with neutropenia by different etiologies may contribute to the construction of educational material for pediatricians, being an auxiliary tool in the early diagnosis of this condition. A retrospective cohort descriptive study was conducted to analyze patients diagnosed with chronic neutropenia in children (o to 21 years of age), followed in the area of Allergy and Pediatric Immunology/ Primary Immunodeficiency at a Terciary Center. Data analysis showed no gender predominance. There was an average time interval of 24 months between the age of onset of symptoms and the age of first consultation. $70.8 \%$ of patients had two or more types of infections. More than half of the patients analyzed $(62.5 \%)$ required hospitalization in at least one infectious episode. Six of 24 patients (25\%) used Granulocyte Colony Stimulating Factor (G-CSF) / Filgastrima. The analysis of patients diagnosed with childhood-onset chronic neutropenia allowed us to observe relevant clinical characteristics, such as age at onset of symptoms, first care and high probability of hospitalizations due to complications. An organized record of these cases would contribute to early diagnosis and improve patients' quality of life.

Keywords: Neutropenia, childhood, pediatric, clinical.

50. CAJAL CELLS AND POST-SURGICAL DISMOTILITY IN HIRSCHSPRUNG DISEASE Juliana de Oliveira Ribeiro, Lucas Rocha Alvarenga Universidade Estadual de Campinas - UNICAMP

OBJECTIVE: To evaluate interstitial cells of Cajal (ICC) number in the proximal ganglionated colon from patients with Hirschsprung's disease (HD) and their dysmotility symptoms after pullthrough operation. METHODS: We revised the files of 24 patients operated for HD from 2001 to 2014 while followed in a medical school hospital. The histological samples corresponding to the proximal ganglionated intestine were selected to ICC count by immunohistochemistry c-Kit. CICs were counted in 10 high-power fields and classified into groups (according Hagger et al, 2000): 1 - 0: sparse, 2 - 3: few, 4 - 8: moderate and > 8 many. RESULTS: Postoperative complaints frequencies were: constipation $(50 \%)$, vomiting $(37.5 \%)$, retentive fecal incontinence $(29.1 \%)$, recurrent abdominal pain $(25 \%)$, abdominal distension (25\%), re-operations (20.8\%), diarrhea (16.6\%) and enterocolitis $(4.1 \%)$. Most patients declared symptoms in the postoperative period, 12 of them were in group sparse, 3 in group few and 4 in group moderate. Five patients referred no symptoms: three in group sparse e two moderate and one patient has lost follow-up. There was no difference in ICC count between symptomatic and asymptomatic patients $(p=0,506)$. CONCLUSION: A low number of ICC was found in $80 \%$ of the proximal ganglionated intestine from studied patients, this finding was not associated to post-operative complaints or with the surgical technique.

Keywords: Hirschsprung disease, megacolon, interstitial cells of Cajal, child.

\section{COCKAYNE SYNDROME TYPE II: CASE REPORT}

João Oliveira Mota, Mariana Ayaka Yamashita

Faculdade de Medicina de Jundiaí

Cockayne Syndrome is a rare genetic disorder with an autosomal recessive inheritance pattern, caused by mutations in the ERCC 8 chromosome 5 and ERCC6 genes on chromosome 10. The syndrome presents in 3 different types, classified as I or A (apparently normal development until the first year of life), II or B (characteristics of the syndrome present from birth) and III or C (mild form, in which the disease phenotype appears only in adulthood). Patients with this syndrome have retarded neuropsychomotor growth and development, photosensitivity, delayed eruption of deciduous teeth, dental caries, hearing loss, congenital cataract, and characteristic parrot beak face. Objective: To present to the health science community the Cockayne Syndrome through a clinical case, in order to disseminate information about this rare and little studied syndrome, so that in the future there will be a better understanding about the clinical management of the disease and its complications. Case report: We present a case report of the patient LBMS, male, 3 years and 5 months, with type II Cockayne Syndrome, presenting with microcephaly, congenital bilateral cataract, delayed neuropsychomotor development, bilateral hearing loss, thoracic tonsillary thorax sharp, upper and lower arch dental caries and "parrot's beak" face. On exome sequencing, the patient presents mutation of the ERCC6 gene with substitution of the amino acid arginine for tryptophan, a variant that has been previously reported in the medical literature associated with cockayne syndrome. Serine amino acid substitution by histidine was also identified, a variant that had never been previously reported in the medical literature.

Key words: syndrome; Cockayne's syndrome; Cockayne Type II

\section{ATYPICAL MANIFESTATIONS IN CHILDREN WITH GUILLAIN BARRÉ SYNDROME}

Julia Brasileiro de Faria Cavalcante, José Edison da Silva Cavalcante, Pedro Nogarotto Cembraneli

Faculdade de Medicina FACERES

INTRODUCTION: Guillain Barré Syndrome (GBS) is an acute single-phase causal disease that occurs after an infection. An analysis ranges from 0.5 to 1.5 / 100,000 children, predominates in males. Initiatives with the limbs followed by progressive, symmetrical muscle weakness, with the principle of lower nodes (lower limbs). In children, the predominance is the difficulty of gait, and greater involvement of the cranial pairs. CASE REPORT: Female patient, one day and one month old, with a history of low fever, blackboard and crying at five days, progressing with progressive loss of strength in asymmetric lower limbs. Found in regular general condition, tachycardic, right upper limb and left lower limb hypotonia, positive safety sign to the right, facial and Achilles stretching, stiff neck stiffness, painful lower back pain in the lower limbs to the xiphoid appendix, being made GBS diagnosis. The cerebrospinal fluid (CSF) did not change and the electroneuromyographic examination showed reduction of muscle action potentials, normal motor conduction velocity, acute motor axonal neuropathy orientation. It evolved with respiratory muscle arrest, requiring intensive care (ICU) therapy and orotracheal intubation. From the immunoglobulin and is in gradual recovery of movements and reflexes. DISCUSSION: Young children are difficult to diagnose because they have atypical complaints and a more challenging neurological examination. In case of suspicion, the patient should be hospitalized in a pediatric ICU, 
where he / she should remain monitored, with frequent surveillance, and treatment should not be delayed, reducing the frequency and severity of complications. Conclusion: It is very important to point out that if you are facing a suspicion of GBS, even if it is atypical, the patient should be hospitalized in a pediatric intensive care unit, where he should be monitored and frequently monitored, providing life support and care. reducing the frequency and severity of complications.

Keywords: Guillain Barré Syndrome; Paresthesia, Asymmetry.

\section{Social Sciences}

53. PROFILE OF THE USE OF ANTIRETROVIRAL REGIMENS IN PATIENTS TREATED AT THE CLINICS HOSPITAL OF STATE UNIVERSITY OF CAMPINAS HIV/AIDS OUTPATIENT CLINIC.

Flávia F. Moreno*, Márcio C. Melo, Carolina N. Bühl, Flávio E. P. Filho, Francisco Aoki, Maria R. Donalisio

Universidade Estadual de Campinas - UNICAMP

Antiretroviral therapy (ART) has contributed to reducing the incidence of opportunistic diseases, hospitalizations, and deaths in patients with HIV/AIDS. The study aims to describe the main medications, treatment regimens, and adverse events in patients who attended the HC-Unicamp HIV/AIDS Outpatient Clinic in 2016. Among the 340 medical records, men were predominant $(61.8 \%)$, the age range from 30 to 49 years old $(57.4 \%)$, heterosexual $(50.9 \%)$, and coming up from the metropolitan region of Campinas $(81.8 \%)$. The most frequent regimens were: TDF + $3 \mathrm{TC}+\mathrm{EFV}$ $(36.8 \%)$ followed by TDF $+3 \mathrm{TC}+\mathrm{ATV} / \mathrm{r}(15.3 \%)$. In $7.7 \%$ of the patients, the scheme contained four or more medications. There were $79(23.2 \%)$ adverse drug effects in 2016 , mainly gastrointestinal (31.4\%), neurological $(27.9 \%)$ and adverse cutaneous drug reaction (17.7\%). This information can contribute to the assessment of care and the impact of that on patient health.

\section{Keywords: AIDS, HIV, Antiretroviral Therapy}

\section{BASIC LIFE SUPPORT TRAINING FOR PUBLIC SCHOOL EMPLOYEES}

Gabriela Acurcio Barbosa, Eduardo Ferreira Sbroggio, Leticia Cristina Francisco, Marília Motta Amelotti, Karin Hayashi, Joaquim Simões Neto Pontifícia Universidade Católica de Campinas

INTRODUCTION: First-time care in a life-threatening situation is critical, and immediate and effective delivery by those on-site, often non-health professionals, is important. However, when properly trained, anyone is able to identify a harmful situation, seek professional support, and correctly perform basic life support (BLS) maneuvers, providing the victim with a higher survival rate and reduced sequelae. OBJECTIVES: To train and educate public high school staff to perform BLS, through measures such as cardiopulmonary resuscitation (CPR) and disengagement maneuvers in adults, children and infants at urgent and emergency situations, until specialized care arrives at the scene. MATERIALS AND METHODS: A six-hou course was held, distributed between theory and practice. Initially, theoretical lecture was given. The interactive activity took place with specific dolls to simulate first aid maneuvers. For the theoretical evaluation, questionnaire was applied covering the stages and maneuvers of the BLS The practical evaluation was done through a return device installed in the dolls, and it was possible to evaluate the hand positioning, frequency and depth of the CPR. Assessments were performed before and after the training to measure the knowledge acquired by participants. The compiled data was organized and a statistical analysis was made through the Student's T-test, with scores between 0 and 100. RESULTS: As a result, the initial theoretical test averaged was $35.8(+21.2)$; at the final test, average was $72.5(+22.8)$ The statistical significance of the theoretical evaluation was $p<0.0001$. In the practical evaluation, initially, the average was $5.78(+14.07)$ and at the final test, the average was $86.5(+12.19)$. The statistical significance of the practical evaluation was $p<0.0001$. DISCUSSION: Every minute a victim does not receive CPR, their chance of survival decreases by about $10 \%$. Thereby, the general objective is to simplify the guideline for $C P R$, in addition to emphasizing the importance of performing it quickly and with high quality at the pre hospital environment. Since 2010, compressions-only CPR has been indicated for lay rescuers, removing the indication of ventilation. This maneuver is easily performed and guided, for instance, by a telephone call. Additionally, the immediate beginning of CPR is indicated for any adult victim, without prior checking of the pulse. BLS training also involves airway clearance maneuvers. Choking may lead to cardiopulmonary arrest, but it is reversible if approached with the Heimlich maneuver - for adults and children - or with backbeats alternating with chest compressions - for infants. The teaching of BLS skills to lay people in Brazil is still extremely neglected. The results obtained in pre-training assessments reflect the population's lack of capacity in identifying emergency situations and how to conduct them until assisted by a health service. CONCLUSION: Performing theoretical and simulated practice guidelines proved to be effective in establishing skills and competencies to perform CPR and disengagement techniques quickly and effectively.

Keywords: Basic Life Support, Simulation, Cardiopulmonary Resuscitation, Heimlich Maneuver, Health Education

\section{EVALUATING THE INFLUENCE OF EATING HABITS IN THE INCIDENCE OF HIGH BLOOD PRESSURE IN PATIENTS OF CLINICS' AMBULATORY FROM EMILIO CARLOS' TEACHING HOSPITAL/ SÃO PAULO}

Priscilla Ferreira Viana Carbone, Ana Luíza Duran Casseb, Giovanna Garcia Marangoni, Durval Ribas-Filho, Durval Ribas-Filho Centro Universitário Padre Albino - FAMECA

INTRODUCTION: Systemic Arterial Hypertension (SAH) is defined as a multifactorial syndrome involving not only higher levels of blood pressure, but it's associated to hormonal or metabolic changings and cardiac hypertrophy. The SAH is also recognized for increasing the risk factor for development of stroke and acute myocardial infarction. Although most of the SAH cases be diagnosed in adulthood, there are some proof that it can be also diagnosed in childhood or during puberty. The effects of a healthy and balanced diet over the blood pressure levels are known, and so is its importance. Among them, the excess in consumption of sodium and sugar will be the key factors addressed in the present study. METHOD: It is a cross sectional incidence study. Through an interview with a group of 37 patients $(\mathrm{N}=37)$ of Clinics' Ambulatory from Emilio Carlos' Teaching Hospital, including 22 females and 15 males, were collected data about the diet, the consumption of sodium and individuals historic of cardiovascular diseases, such as the incidence of Systemic Arterial Hypertension cases. RESULTS: The consumption of straightforward carbs and sugar-sweetened beverages (sodas and processed juices) were elevated, such as and the excess ingestion of processed foods, which includes a huge amount of sodium, were observed. When it comes to fractioning the meals during the day long, $43,2 \%$ of the group of patients studied doesn't take a snack in the middle of the morning - between breakfast and lunch - $32,4 \%$ doesn't take the afternoon's snack and 54\%, the dinner. Discussion: Authors observed that the large daily intake of straightforward carbs, caster sugar, processed foods - sources of sodium -, and sugar-sweetened beverages attached to the lower incidence of physical exercises regularly, confirm the high prevalence of patients with SAH and other cardiovascular diseases. Therefore, a balanced diet associated with regular physical exercise to reduce de incidence of SAH and comorbidities. CONCLUSIONS: Dietary habits shows that $89,2 \%$ of the patients have SAH, which $86,5 \%$ of those used to seasoning the salad with salt, while $13,3 \%$ doesn't use salt to do the same. In relation to the main meals, $53,3 \%$ of the patients with $\mathrm{SAH}$ uses industrialized seasoning to prepare the meal.

Keywords: Systemic Arterial Hypertension, dietary habits, sodium levels, cardiovascular diseases, processed foods.

56. COMMITMENT OF HEALTH TEACHING TO THE UNIFIED HEALTH SYSTEM PERCEPTION OF GRADUATES IN THE INSTITUTIONAL PROGRAM FOR TEACHING INITIATION SCHOLARSHIPS - NURSING

Luana Cristina Hencklein, Mara Regina Lemes de Sordi Universidade Estadual de Campinas - UNICAMP

The Unified Health System provides guarantee of full, universal and free access to the entire population of Brazil, whose comprehensive health care has become a right of all Brazilians. Given the numerical expression of nursing technicians in the provision of health care, it is justified that the ways of training these workers are based on these principles and compose the pedagogical project of schools that are responsible for training. Thus, the teaching in technical professional education of secondary level in Nursing is of fundamental relevance for the production of health care consistent with the principles of Unified Health System. This study aims to know and to discuss the perceptions of the graduates of the Program for Teaching Initiation Scholarships-Nursing subproject developed at State University of Campinas. It is a program aimed at the initiation of teaching and involves the repercussions of the lived experience, taking as reference 
the guiding axis of the program that is intended to discuss the teaching work with future mid-level technical nursing workers and its implication with the Unified Health System. The methodology consisted of a qualitative study involving pedagogical narratives of graduates who participated in Program for Teaching Initiation Scholarships-Nursing for at least 6 months. The obtained data, treated according to Bardin, generated four categories: the nurse's commitment to the nursing technician's formation and its interface with the Unified Health System; the educational role of the nurse; the teacher's performance in the School's Pedagogical Political Project and the relationship between bachelor degree and college degree of nursing at State University of Campinas. The conclusions of the study reveals that this program in nursing, according to the project's guiding axis, was an indispensable experience to strengthen the senses of a health teaching referenced in Unified Health System principles and implied with the valorization of mid-level technical workers, subsidizing the construction of a teaching identity based on formative actions committed to the right of health and of pedagogical practices based on a humanized, critical and less technical approach. It was confirmed that pedagogical narratives are a potent resource for important reflections to redefine the educational role of nurses.

Keywords: Nursing; Pedagogical narratives; Unified Health System; Teacher training.

57. ANALYSIS OF VIOLENCE IN THE SCHOOL BEHAVIOR OF 4th AND 5th GRADE STUDENTS OF A LOCAL SCHOOL IN A SMALL TOWN IN THE INTERIOR OF SÃO PAULO STATE, BRAZIL

Lana Maria Freitas Alves de Oliveira, Luma Comes Lourencini, Heloísa Fusetto de Lima, Beatriz Paterno Brito, Geovana Caliente Marques, Uderlei Doniseti Silveira Covizzi

Centro Universitário de Votuporanga

The school's main mission is the development of teaching-learning processes, playing a fundamental role in the formation and performance of citizens in all areas of social life. The school environment applies a heavy weight on the social development of human beings, directly influencing their behaviors, which can be translated into externalizing factors, directly affecting society and the environment in which they are inserted, and internalizing ones, which are reflected in the individual. The study aimed to analyze the influence of this environment on the education and social health of 4 th and 5 th grade students of local school in a small town in the interior of são paulo state, brazil. The method was based on playful activities with the purpose of bonding and subsequent application of the questionnaires component of the Inventário de Habilidades Sociais, Problemas de Comportamento e Competência Acadêmica (SSRS) for students, their guardians and teachers, which was used as an instrument. Screening by analyzing the responses of parents, teachers and the child, it was possible to assess the repertoire of social skills and indicators of children's behavior and academic competence problems. From the results obtained, we proceeded to the quantitative evaluation, relating events of violence with the school environment. Finally, a direct influence of the school environment on the students' development and interpersonal behavior was evaluated as well as the close relationship with violent attitudes in the in-school relationship of the citizen in formation. The analyzed children practice greater verbal violence and showed awareness regarding the consequences of these violent acts to the physical and psychic to both the victim and the aggressor. The children's perception of an essential adult role in resolving these conflicts was notorious. Concerning SSRS, it is concluded that most students need social skills training, especially for social and academic adjustment, which is fundamental to ensure so-called school health, after all health and education should be offered in full to assist full enjoyment of human rights. However, children have the right to an education that aims at the full development of their person, their preparation for the exercise of citizenship and their qualification for work, and promoting the culture of peace is a prevention of health problems, whether physical or mental.

\section{ASSESSMENT OF PRYMARY HEALTH CARE: HEALTH PROFESSIONAL'S} PERSPECTIVE

Isabelle Sarpa, Isabella de Oliveira Fadoni, Gabrielle Karen Cardoso Villena, Mariane de Oliveira Diogo Scussel, Felipe Vilela da Costa, Maria Luíza Rennó Moreira Baldassaris

Universidade do Vale do Sapucaí - UNIVÁS

JUSTIFICATION :The full operation of attributes of the Family Health Strategy (ESF) e consequently of the Primary Health Care (APS) bring to the service a satisfactory workflow. The assessment of ESF is one task that require a participation of differents institutions and professionals in a collective, continuing and persevering effort. For this reason, the interest in using a tool to evaluate the service was raised. Objective:The study aimed to evaluate the professionals of 15 Team of ESF from Pouso Alegre- MG with the objective of detect any potential problems wich reduce the effectiveness of ESF and develop actions to improve the quality of service afterwards. METHODS: For this analysis, the Primary Care Assesment Tool - PCA Tool professional version was used. This tool aims to assess the degree of orientation of APS through the following attributes: Accessibility, Longitudinality, Coordination, Completeness, Family and Community Counselling. RESULTS: The partial results was satisfactory: all percentage attributes stayed above $60 \%$. The attribute with the best evaluate was Coordination - Information System and the worst evaluate was Community Counselling. CONCLUSION: For finals results, it still necessary the full statistical analisys from the collected data.

Keywords: Prymary Health Care, Assessment the Quality of Health Care, Health Care Quality, Health professional's, Public Health.

\section{THE IMPORTANCE OF LEARNING LIBRAS FOR DOCTORS}

Gabriela Maris Bitencourt da Rocha, Ana Claudia Balieiro Lodi, Grupo PET, Marcelo Riberto

Faculdade de Medicina de Ribeirão Preto - USP/RP

The term hearing impairment is linked to hearing loss, but also to the communication barriers faced by deaf people to participate in society. Currently, the main barrier has been the lack of knowledge and / or little recognition of the Brazilian Sign Language (Libras) in different social spaces, especially those of health care, a fact that materializes in the lack of translators-interpreters of Libras - Portuguese (TILSP) in these places.The presence of these professionals, when existing, results from the investment of the deaf themselves, a condition faced by approximately $1.1 \%$ of the Brazilian population. The lack of knowledge of Libras by health professionals and the absence of TILSP ends up promoting written-deaf medical communication, which has limitations that may contribute to misdiagnosis and non-adherence to treatment, as well as the lack of care, since it is necessary to establish effective communication between doctor and patient. Thus, the importance of learning Libras for doctors and students in the area, focused on situations and vocabulary of clinical interest, is highlighted.

\section{VACCINATION AGAINST HPV IN BRAZIL: ADHESION CHALLENGES}

Laís Beluci Camargo, Isabel Chaltein Thomazella Ribeiro, Lúcio Fábio Caldas Ferraz

Universidade São Francisco

INTRODUCTION: The infection by the human papillomavirus (HPV) is a sexually transmitted disease of highest prevalence in Brazil and in the world. The HPV has a number of manifestations due to its genotypic variety, its most common and benign manifestation is the genital warts, however, the existence of oncogenic genotypes as HPV 16 and 18 link the infection to many neoplasms in men and women. Between the neoplasms,cervical cancer is the most important one and,due to its high prevalence and mortality among women,in Brazil it is considered the fourth cause of death by câncer. Due to this, the quadrivalent vaccine against the HPV was added to the National Calendar of Vaccination in 2014 targeting women of 9 to 13 years old age group, and afterwards in 2017, men of 11 to 14 years old age group weer added to the program. However, the adhesion to the vaccine was below expectations, especially to the second dose and among males, which in turn prejudices the effectiveness of the immunization. In this sense, this study aimed to investigate and to understand the main barriers to the adhesion of the vaccine that contributes to the maintenance of the high morbimortality of the HPV infection. METHODOLOGY: Selection of articles published between 2013 to 2017 in online databases that had any of the following descriptors: Human Papilloma Virus, Papillomavirus Vaccines, Uterine Cervical Neoplasms. The only exception to the rule was an article dating from 2009 that explained the relation between HPV infection and cervical cancer. It was also added official information by the Brazilian Ministry of Health about its immunization program, dating since the implementation in 2014, to more actual data of 2018. RESULTS: The main reasons for low-adhesion are due to doubts about the effectiveness and the safety of the vaccine, due to taboos regarding sexuality, and mostly, due to the lack of knowledge, in adolescents and parents, about the HPV, its transmission and impacts. DISCUSSION: Since studies prove the effectiveness of the vaccine in the prevention of HPV manifestations, especially cancers, and endorse its safety meaning that it does not cause 
adverse effects damaging to health, and prove that there is no relation between the vaccine and risky sexual practices, it is evident that there is a difficulty to transmit this health knowledge to the population in a way to validate the implementation of the immunization program. CONCLUSION: The challenges that the health organizations in Brazil face to prevent the disease using the vaccine can be overcome by the development of strategies to increase the knowledge of the population through a combined approach with the educational institutions and the media, focusing on the points of fragmentation of knowledge about the vaccine and HPV, as well as desmitifications of sexuality taboos in order to increase the vaccination acceptance.

61. UNIVERSITY LIFE, ACADEMIC CULTURE AND HEALTH: SEMIOTIC ANALYSIS OF IMAGES CARRIED OUT IN A CAMPUS

Fernanda Dermando Brida, Bruno José Barcellos Fontanella

Universidade Federal de São Carlos

This research paper aimed to interpretate advertising images that were part of posters and banners attached around a Brazilian public university campus, frequented by about 8500 graduate and 3300 post-graduate students. From 2018 February to 2019 May, these kind of images were collected/photographed in varied campus locations. In this paper, banners that advertises universtity parties were excluded, as they were analysed and discussed in a previous paper. The images' plastic, iconic and linguistic elements underwent a semiotics analysis, that generated 84 themes, from 146 banners, organized in six thematic categories: "Primary and secundary needs and other vulnerabilities", "Academic needs mentions", "Atributes and qualities of the advertisements", "College life acculturation", "Estructural and future life issues" and "Especific themes approached in college residence hall". Products and services were offered as easyeffective-economic-fasted solutions, generally not associated to health promotion mentions, in spite of the references to nutrition and physical activities. Solutions for alleged educational gaps were commonly offered by private companies, such as English language schools. Cultural, religious, entertainmental and political activities were also offered. In the university residence hall, the banners approached specially ideas, instead of products, for example: violence against women, student rights, environment and sustainability and physical and mental health issues. The banners and posters were attached in places that receive great student flow and probably have reached great number of students. Several marketing initiatives are inserted in the campus informally, not necessarily - and generally not - propagating values, products, and ideas tied to university's policies about pedagogical assistance or students' health promotion, prevention and health care.

Keywords: student health; university campus; advertising; qualitative resarch; semiotic analysis.

62. THE USE OF TECHNOLOGY IN TEACHING CARDIOPULMONARY REANIMATION

Alexandra Mauriel dos Reis, Beatriz Carvalho de Campos Bueno, Daniella Moretti Arbore, Gustavo Rodrigues de Paula, Vitor Duarte Moron de Andrade, Joaquim Simões Neto Pontifícia Universidade Católica de Campinas

Cardiopulmonary reanimation (CPR) consists of a set of maneuvers that ensure tissue oxygenation when blood circulation is stopped. The project seeks to verify the employees training effectiveness in the area of education to perform the CPR maneuver, providing an efficient and immediate first care, increasing the chances of survival. Bluetooth-equipped mannequins connected to a mobile application were used for the training and also to extract information on the effectiveness of CPR before and after theoretical and practical classes. Initially the effectiveness was $17.96 \%$ and after training was $79.03 \%$, a significant increase. CPR training for education staff contributes to increase the chances of survival of cardiac arrest patients by the effectiveness of the maneuver.

63. QUALITY OF LIFE OF FAMILIES WHO HAVE CHILDREN WITH EPIDERMOLYSIS BULLOSA IN BRAZIL

Heloísa Pastana Marsiglio, Sandra Mara Bispo Sousa, Débora Gusmão Melo

Universidade Federal de São Carlos

Epidermolysis bullosa (EB) encompasses a group of rare genetic diseases with an estimated incidence of 19:1.000.000 live births. It's types and subtypes are caused by mutations in several genes, which encode proteins involved in skin's mechanical support. Because of that patients with EB have fragile skin, resulting in blistering and lesions. This research investigated the impact of $E B$ on the family quality of life (FQOL). This is a descriptive and cross-sectional research that has developed with the formal support of the DEBRA-Brazil (Dystrophic Epidermolysis Bullosa Research Association). This study included 113 families who have children with EB linked to DEBRA-Brazil, aged between 2 months and 60 years. Data were collected by questionnaires including sociodemographic information, profile of the person with EB, Barthel and Lawton C Brody functional indexes, and the Beach Center Family Quality of Life Scale. Individuals with EB had an average score of $69.38 \pm 30.33$ in the Barthel index, indicating moderate dependence on basic activities of daily living; and an average score of $3.31 \pm 2.93$ on the Lawton a Brody index, indicating severe dependence on instrumental activities of daily living. The average score of total FQoL $(3.857 \pm 0.505)$ was lower than the scores of the "family interaction" domain (4.069 $\pm 0.654 ;$ p $<0.001)$. "parenting" domain $(4.030 \pm 0.585 ; \mathrm{p}<0.001)$ and "disability-related support" domain $(4.055 \pm 0.611 ; p<0.001)$, and higher than

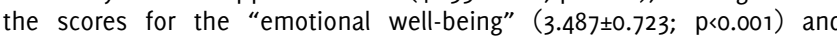
"physical-material well-being" $(3.533 \pm 0.730 ; p<0.001)$ domains. The multiple correlation showed that interference with interpersonal relationships, the Barthel's functional index, multiprofessional health monitoring, family income, gender of the person with EB and the main feeling about the disease were predictors of total FQoL. The coefficient of determination for this model was $R^{2}=0.472$, indicating that the model has explained $47.2 \%$ of the variability found in the total $F Q 0 L$ results $(p<0.001)$. These results may contribute to the construction of a comprehensive care line for patients with EB and their families in the Brazilian National Brazilian Health System.

Keywords: Epidermolysis bullosa, rare disease, family quality of life, family relations.

\section{SURVEY OF ADOLESCENT HEALTH NEEDS: AN ANALYSIS CARRIED OUT AT} THE EDUCATIONAL INSTITUTION OF A CITY IN NOROESTE PAULISTA

Vinicius Cavallari, Natália de Oliveira Geretti, Bianca Cardiano, Lívia Duarte Olher, Camila Oliveira de Castro, Marinês Ralho Unifev - Centro Universitário de Votuporanga

This work was developed to raise the needs of adolescents from an established territory for the development of activities. In order to guide the adolescent in this transition phase characterized by biopsychosocial transformations and conflicts of relationships with parents and the environment in which they live, activities were developed that introduced several issues related to sexually transmitted infections (STIS), sexuality, personal hygiene, life perspective, equity and promotion of public health. The work started from a visit to UBS, to survey the projects developed with adolescents in the considered territory. From the experiences acquired by the group, it is worth all the topics studied, since they provided insight into how adolescents may be inserted in a risky and vulnerable environment for their biopsychosocial development.

Keywords: health needs, adolescents, vulnerability, public health

\section{THE IMPORTANCE OF BOND BUILDING FOR DEVELOPMENT AND} COMPLETENESS IN MEDICAL PRACTICE: AN EXPERIENCE REPORT

Lucas Ajonas Alves, Brenda Moreira Benites, Ana Beatriz Ferreira Mendes, Isabela Guarnieri Borges, Victória França Coelho, Marinês Ralho

Unifev - Centro Universitário de Votuporanga

The exposed work was developed with the aim of establishing affective bond with adolescents from a city of Noroeste Paulista, place established for the development of this study. Bond is more than just contact, it means the establishment of a continuous relationship in time, personal and nontransferable, meeting subjectivities. This work was directed by the National Policy of Primary Care, the Basic Guidelines of Comprehensive Health Care for Adolescents in Schools and Basic Health Units, the Adolescent Health Program (PROSAD). It also was guided by the laws governing the Unified Health System (SUS) - Law 8.080/90, which corroborates as one of its fundamental principles. Through contact with professionals of the Basic Health Unit (UBS) it was possible to see a growing demand of adolescents who need a specific work for health prevention. This practice has allowed to create bond.

Keywords: bond, adolescence, equity, integrality, public health 
66. SEMIOTIC ANALYSIS OF DIVULGED IMAGES BY PRIMARY HEALTH CARE SERVICES OF SÃO CARLOS - SP

Nicolas Cabral de Andrade, Bruno José Barcellos Fontanella Universidade Federal de São Carlos

This research work aimed to interpret the images of advertisements on posters in primary health care units in the city of São Carlos (SP). It was sought to analyze the extent to which, in these materials, symbolism would be used that results in certain conceptions about health and health care, conveying or evoking certain values and understandings about the biopsychosocial and cultural aspects involved. Between May 2018 and April 2019, 80 posters were photographed, their plastic, iconic and linguistic elements subjected to semiotic image analysis, and seventy-nine themes were analyzed and organized into four thematic categories. The conclusion reached is that unofficial actions of informal health marketing, having as enunciators especially the local health teams, seem to propagate ideas and values about conceptions of the health-disease-care process, not necessarily corresponding to educational goals and methods ratified by the knowledge area of collective health.

Keywords: Health Education. Marketing of Health Services. Primary Health Care. Semiotics. Public Health.

\section{YeLLOW FEVER: ALTERATION OF EPIDEMIOLOGICAL PATTERN AND} CONSEQUENCES TO UNUSUAL BRAZILIAN REGIONS

Meg Thaynara de Moura Moreira, Marcela Bulkool Aquino, Marcella Silva Borges, Paula Sommer, Roberta de Mattos Figueiredo

Centro Universitário Estácio de Ribeirão Preto

Yellow Fever, it's originally from tropical Brazilian regions as a result of vector cycle. However, it has been geographically disseminated to other areas in the country as well, such as South and Southeast regions. The main goal of this work is to create a bibliographic survey to check specific data and dissemination reasons for such unusual propagation to non tropical regions. The chosen Methodology was articles reviews on scientific platforms and also websites, including DATASUS, FIOCRUZ and Ministério da Saúde to epidemiological data collection. The results indicate that the reasons for such unsual dissemination was climate changes, natural disasters, urban mobility and different niche. Therefore, it is necessary to bring up new protective and preventive strategies to the population, aiming at healthy promotion and outbreaks combat. In addition, population must be oriented during season and pre-season period, when vector cycle is favored. Added to such measures, structuring protective programs to preserved areas is extremely important to avoid displacement of yellow fever virus to urban environment and reinstallation of urban yellow fever in the country.

Keywords: yellow fever, epidemic, endemic regions, Brazil

\section{IMPACT ON STUDENTS AFTER INITIATION OF A PALLIATIVE CARE ROTATION} IN THE CURRICULAR GRADE OF STUDENTS OF MEDICINE IN BRAZIL

Carolina Neves Bühl, Cristina Bueno Terzi Coelho, Jussara de Lima e Souza, Daniele Pompei Sacardo, Flávio César de Sá Universidade Estadual de Campinas - UNICAMP

BACKGROUND: Brazil has one million deaths a year, 650 thousand of them due to chronic diseases, thus patients eligible to PC. However, there is still a gap in the training of physicians and health professionals on this topic. Therefore, an educational change is necessary to cover the national health system with appropriate PC services. RESEARCH OBJECTIVES: To compare the perception of the students in regards to the importance of PC for patients and the role of doctors on this topic before and after the introduction of the PC course in the curriculum of undergraduate medical students. METHODS: Medical school in our University has a 6-year duration. The PC course was implemented for 4 th year students in 2018. The program encompassed 4 hours of lectures, 12 hours of bedside teaching at the neonatology, oncology and internal medicine rounds and 12 hours of supervised patient home visits. A questionnaire was applied to assess pre- and post-course perception of the students in regards to several aspects of PC. The students were required to choose one of the options: strongly agree, agree, neutral, disagree or strongly disagree to the following statements: a) primary care doctors play an important role in PC services; b) It would be helpful for me to receive further training in PC; $c$ ) doctors play an important role in the spiritual support for patients facing end-of-life diseases; $d$ ) I believe that $P C$ service improves the overall standard of care. The Chi-square test was used for statistical analysis of the data at $P<0.05$. RESULTS: Overall 106 questionnaires were responded ( 55 pre-course and 51 postcourse). The perception of the students in regards to the statements "a", "c" and "d" shifted from "agree" to "strongly agree" after the conclusion of the course $(P<0.05)$. There was no difference in regards to statement " $b$ " as the majority of the students strongly agreed with such statement before and after the course $(P>0.05)$. CONCLUSION: The perception of the students that further training in PC would benefit their skills was present before and after the course. On the other hand, the students realized that primary care doctors play an important role in PC service. Their perception that doctors can contribute for the spiritual care of patients and families increased, as well as the perception that PC is beneficial for patients. IMPLICATIONS FOR RESEARCH, POLICY, OR PRACTICE: Overall the students reported enhanced perception of the importance of PC. Our course seems to be suitable for implementation in other medical schools in countries with the same level of education as ours.

\section{Surgery}

\section{ACUTE COMPARTIMENTAL SYNDROME IN UPPER MEMBER: REPORT OF} TWO CASES AFTER CATHETERIZATION

Isabella Casani Rech, Otacílio de Camargo Junior

Pontifícia Universidade Católica de Campinas

INTRODUCTION: Acute compartment syndrome (ACS) is a sudden increase in pressure in a restricted anatomical space, with a decrease in blood perfusion of the muscles and organs contained in it, causing ischemia and tissue necrosis if not reversed early. The treatment consists of performing fasciotomy, which is defined by a surgical opening of the compartments for decompression. It is characterized by paraesthesia, continuous pain, hypoesthesia, edema and stiffening of the affected region. CASE REPORTS: CASE 1: A 66-year-old female patient underwent curative cardiac catheterization with stent implantation in the anterior descending coronary artery $(A D)$. Nine hours after the procedure, the patient is reevaluated for compartmental syndrome in MSD. Performed eco-color-Doppler on MSD. opted for fasciotomy and arterial surgical exploration. In the surgical procedure, radial artery pseudoaneurysm was observed, with active and jet bleeding, after removal of the local hematoma. Arterrhaphy, hematoma drainage, fasciotomy with immediate restoration of distal flow, perfusion and temperature. CASE 2: A 78-year-old male patient underwent cardiac catheterization for unstable angina with angioplasty in AD. Twenty-four hours later, it is requested to reevaluate the patient for pain, edema and hematoma in the limb. US performed with brachial artery pseudoaneurysm diagnosis. Submitted to rafia with good postoperative evolution. DISCUSSION: Acute compartment syndrome is a surgical emergency and its occurrence after cardiac catheterization in the upper limb is infrequent, but should be suspected whenever the patient presents with ischemic syndrome.

\section{DERMATOLOGICAL MANIFESTATIONS ON POST-BARIATRIC SURGERY:} SISTEMATIC REVIEW ON LITERATURE

Andressa Christine Ferreira Silva, Laura Moya Kazmarek, Elemir Macedo de Souza Maria Letícia Cintra

Universidade Estadual de Campinas - UNICAMP

Obesity and overweight are among the world's largest public health concerns. As obesity increases, so does the number of bariatric surgeries, considered effective treatments for obesity and comorbidities. Among the main complications after bariatric surgery are nutritional deficiencies, which may be due to heavy weight loss, nutrient malabsorption and/or change in habits. The most common deficiencies are those of vitamin B12, iron and folate and eventually manifest through dermatological changes, which may progress to severe disorders. Knowing and identifying them promptly may prevent systemic damage. We selected from the literature all articles available on short, medium and long-term skin manifestations related to post-bariatric nutritional disorders of any category; we identified the most frequent manifestations and which nutritional deficiencies they are associated with. The research was carried out in health research portals and databases of national and international biomedical journals (PubMed, Virtual Health Library (VHL/Biblioteca Virtual em Saúde - BVS, in portuguese) Regional Portal, Scopus, EMBASE, Web of Science and Scielo), without limitation on Publication. The following descriptors were used for the research, following the ideal methodology in each database/research portal: "bariatric surgery", "skin", "skin disease", "skin manifestation", "deficiency disease", "malnutrition". Exploratory data analysis was performed by summary measures. To assess the association between the 
variables, a meta-analysis for individual data (IPD meta-analysis) was performed by the Laplace method of mixed-effect logistic regression. The adopted significance level was $5 \%$. We obtained 53 articles, which 22 of them being from literature review, discussion of the theme or research on specific dermatological manifestations, and 31 articles reporting dermatological manifestations after bariatric surgery, totaling 35 articles. The average age of the patients was 44 years and the average time between surgery and dermatological manifestation was 5 years. In these patients, the most common type of surgery was Roux-en-Y gastric bypass $(68 \%)$, followed by biliopancreatic shunt $(14.29 \%)$ and several other types. Among the articles in which contained information, patients adherence to postoperative nutritional supplementation, only $50 \%$ of them adhered. The most identified deficiencies were zinc $(60 \%)$; vitamin A $(40 \%)$; vitamin D (28\%); copper (25\%); vitamin B3 and vitamin E (11\% each); selenium and iron ( $8 \%$ ); calcium, vitamin $\mathrm{K}$ and vitamin C (2 to $5 \%$ each). Among the manifestations, $28 \%$ was enteropathic acrodermatitis, which was significantly associated with the RYGP type $(P<0.001)$ and zinc deficiency $(P$ $<0.001$ ), unrelated to the postoperative time interval. We found a significant association between enteropathic acrodermatitis, zinc deficiency and Rouxen-Y gastric bypass (RYCP) technique. RYGP was the most common bariatric surgery in this sample, supporting that the data indicate this is the most commonly performed type of surgery.

\section{Keywords: bariatric surgery, skin manifestation, deficiency disease}

\section{COMPARTIMENTAL SYNDROME AFTER ARTERY-VENOUS FISTULA: A CASE} REPORT

Lina Celescuekci, Lorena Murta dos Santos, Otacílio de Camargo Junior Pontifícia Universidade Católica de Campinas

Upper limb artery-venous fistulas (AVF) are definitive vascular accesses, performed either therapeutically for hemodialysis or prophylactically for future hemodialysis sessions. Since high arterial flow moves through the low-resistance conduit and high venous compliance, the patient with AVF evolves with arterialization of the venous segment and the formation of single or multiple aneurysms in the venous body of AVF. Compartment syndrome is the increase of pressure in a restricted anatomical space with decreased blood perfusion of the muscles and organs contained within it, which may cause tissue damage and necrosis. The treatment consists of performing fasciotomy, a surgical opening of the compartments, to relieve internal pressure and thus restore blood circulation to the tissues, resulting in an operative wound. Characterized by paresthesia, continuous pain, hypoesthesia, edema and stiffening of the affected region. The main causes may be limb constriction by plaster, compressive dressings, and a possible increase in substances in the muscle compartment caused by edema or hemorrhage. When it comes to hemorrhage, the surgical treatment beyond fasciotomy should also contain this hemorrhagic lesion. CASE REPORT: A 58year-old male patient underwent basilic-radial arteriovenous fistula under local anesthesia and was discharged in the immediate postoperative period. Two days after the procedure, the patient came to the hospital's emergency room reporting severe pain and severe limb edema. On physical examination, he presented severe edema with decreased hand perfusion. $\mathrm{He}$ was submitted to limb fascitomy, hematoma drainage and AVF evaluation that no longer presented active bleeding. Patient evolved well postoperatively. DISCUSSION: Compartment syndrome after artery-venous fistula in the upper limb is not a frequent complication; however, it should be suspected whenever the patient presents with severe pain and edema, possibly with limb ischemia.

\section{OVARY VEIN THROMBOSIS: CASE REPORT}

Laisy Beatris Dolfini Celim, Débora Penna Chaves Bertazzo Otacílio de Camargo Junior

Pontifícia Universidade Católica de Campinas

Ovarian vein thrombosis is a well-known but rare entity that can occur during the postpartum period. This condition has also been associated with inflammatory and malignant processes within the pelvis. Untreated, complications of ovarian vein thrombosis may be significant due to sepsis and the risk of pulmonary embolism. Diagnosis can be reliably made using ultrasound, computed tomography or magnetic resonance imaging. The treatment of ovarian vein thrombosis is particularly important in postpartum patients, and anticoagulant therapy is currently recommended. This paper aims to report the case of a patient who, in her postpartum period, evolved to a urinary tract, which was initially diagnosed with endometritis and pyelonephritis on the left. After a second investigation, through laboratory and imaging exams, thrombosis of the entire length of the right ovarian vein was evidenced. Treated with anticoagulants, it has evolved clinically well. Therefore, despite being rare and with significant complications, ovarian vein thrombosis can be reliably diagnosed by imaging exams: ultrasound, computed tomography and magnetic resonance imaging. In addition, the treatment generates great chances of favorable evolution

\section{Keywords: Thrombosis; ovarin vein; anticoagulant}

\section{AMYLASE DOSAGE IN ABDOMINAL DRAIN CONTENT ON DAY 1 POST-} PANCREATECTOMY AS A PREDICTOR OF PANCREATIC FISTULA

Pablo Henrique Brito da Rosa, Adriana Sañudo, Edson José Lobo, Alberto Condenberg, Gaspar de Jesus Lopes Filho, Franz Robert Apodaca Torrez

Escola Paulista de Medicina - Universidade Federal de São Paulo

INTRODUCTION: Pancreatic neoplasms have as standard treatment pancreatic resection surgery, an invasive procedure of great morbidity and mortality, whose main postoperative complication is Pancreatic Fistula (PF). There is currently a discussion in the literature about what would be the cut-off point for abdominal drain amylase dosage on the first postoperative day (ADA1P0), by which the diagnosis of PF could be excluded. OBJECTIVES Analyze the $A D A 1 P 0$ and evaluate its predictive character in the exclusion of the diagnosis of PF with clinical repercussion, trying to establish a cutoff point from which lower values demonstrate safety demonstrate safety to exclude the diagnosis of PF. MATERIALS AND METHODS: From August 2017 to February 2019, data was collected and 35 patients in a prospective nonrandomized cohort undergoing pancreatic resection were analyzed. The patients were divided into 2 groups, one group consisting of patients who developed PF (Group B) and the other composed of patients who did not develop PF (Group A). The Receiver Operating Characteristic Curve (ROC Curve) was constructed to assess the predictive component of ADA1PO exclusion of the diagnosis of PF. In this graph, cutoff points were evaluated by calculating sensitivity, negative predictive value (NPV) and clinical applicability. The cut-off point selected was the one with the highest sensitivity and NPV associated with the best clinical utility. PARCIAL RESULTS: Group A comprised 22 patients $(62.8 \%), 18(51.4 \%)$ did not evolve with PF and $4(11.4 \%)$ evolved with biochemical leak without clinical repercussion. Group B comprised 13 patients $(37.2 \%), 8(22.9 \%)$ evolved with PF grade B and $5(14.3 \%)$ evolved with PF grade C. Group B had the largest number of days hospitalized and the largest number of days drained, with medians of 26.5 and 28 days respectively, while in group $A$ the medians were respectively 12 and 11 days $(p<0.05)$. The $444 \mathrm{U} / \mathrm{L}$ value was the most satisfactory ROC curve cut-off point ( $\mathrm{Cl}: 0.598-0.898)$, as it presented sensitivity and NPV of $92.3 \%$. This cutoff point was able to select $13(59.1 \%)$ of the 22 patients who did not develop PF. However, 1 patient who evolved with PF grade $C$ had $A D A 1 P O$ lower than $444 \mathrm{U} / \mathrm{L}$, because of this, sensitivity and NPV did not reach $100 \%$. CONCLUSION: The ADA1PO can be used as a predictive factor in the diagnostic exclusion of PF because the ADA1PO ROC curve did not cross the nullity line (IC: $0.598-0.898$ ), with the value of 444 $\mathrm{U} / \mathrm{L}$ being the best performance cutoff point, because it detected $60 \%$ of patients who did not develop PF, besides presenting high sensitivity and NPV.

\section{ABDOMINAL AORTA DISSECTION: REPORT OF THREE CASES}

Débora Penna Chaves Bertazzo, Laisy Beatris Dolfini Celim, Otacílio de Camargo Júnior, Bruna Madeleine da Silva Simplício Flôr, Otacílio de Camargo Junior

Pontifícia Universidade Católica de Campinas

The aortic dissection is defined as the rupture of the intimal layer of the aorta, with consequent separation of the middle layer into two channels, resulting in false lumen within the artery wall. Such disease can affect both thoracic and abdominal aorta. Abdominal aortic dissection is less common and has varied clinical manifestations, with abdominal or low back pain and lower limb ischemia being the most frequent. In this study, we present three cases of infra-renal abdominal aortic dissection in female patients. Despite variations in pain location and duration, all patients had abdominal pain and arterial hypertension. Two patients underwent surgical treatment with good evolution. Because of its rarity, there is little experience in treating infra-renal abdominal aortic dissection, which may be surgical or conservative. At the time of therapeutic decision, consideration should be given to the presence of aortic rupture, lower limb ischemia, intractable pain, progression to aneurysm and false lumen degeneration for the indication of surgery.

Keywords: Dissection; abdominal aorta; abdominal pain. 
75.

\section{AND ITS REPERCUSSION IN PATIENT SAFETY}

Pedro Henrique Alves Silva, Renan Parise Maltempi, Pedro Favero

Martinasso, Murilo Baracat Cortese Conde, João Cesar Jacon

Faculdade de Medicina de Catanduva

Reports concerning patient safety has been related thousands of years ago and these reports stem from the many mistakes made by health professionals. Nowadays, despite the technological improvement in procedures involving surgeries, flaws continue existing in the surgical environment. Even the simplest processes present countless critical steps. Within this context, even after the introduction of the Safe Surgery Challenges established by the World Health Organization (WHO) and the Safe Surgery Protocol proposed by Agencia Nacional de Vigilancia Sanitaria (ANVISA), surgical complications account for a large proportion of preventable deaths and medical injuries. In the context of medical technological advance, another factor that contributes to the occurrence of incidents in the surgical environment is the belief that physicians are infallible, causing negligence of the procedures in patient safety. The main focus of the research was to identify the knowledge and adhesion to the Safe Surgery Protocol by surgeons, as well as the incidence of adverse events related to surgery, besides the knowledge in the matter of the protocol by the own patients. The research consisted in a longitudinal prospective study, with quantitative character. Data collection occurred with an instrument designed for data collection, which gathered the sociographic profile of sixty-eight surgeons and residents, the knowledge and adhesion of those to the safe surgery protocol, and evaluated eightytwo patients, as well as the surgery environment. The results obtained, on the part of the surgeons, showed that despite the contact with the protocol obtained during the graduation period, there was a deficiency in its adhesion, causing adverse events such as the use of uncalibrated equipment. On the part of the patients, these demonstrated the knowledge in terms of the surgery they were about to perform, although many had not been questioned about allergies neither introduced to the surgical staff. Therefore, there were flaws in dynamicity and adhesion in some steps of the protocol, harming the laterality in the process and patient safety.

PERSPECTIVES OF SURGICAL AND PROGNOSTIC MANAGEMENT IN TRANSFERING NERVES TO UPPER MEMBERS - BIBLIOGRAPHIC REVIEW

Rafael Fumachi Bredariol, Mateus Comes Pereira, Gabriel Rocha Sanches Carlos Tadeu Parisi de Oliveira

Universidade São Francisco

INTRODUCTION: Nerve damage can occur with realative frequency in cases of traumas, often these lesions preclude direct or indirect repair, being possible to use the technique of nerve transfer, which is a versatile reconstructive technique mainly used for severe proximal lesions, in which it is usually the only viable option. OBJECTIVE: To review the principles, techniques and results related to the transfer of nerves in the upper limbs, specifically, shoulder, elbow and wrist. METHODS: this is a review centered on data indexed in the virtual platforms: MEDLINE, PubMed, SCIELO and Cochrane, published between the years 2000 and 2017 referring to the topic of nerve transfer in upper limb musculature. DISCUSSION: In this neurosurgical procedure a nerve, usually of minor importance, is disconnected from its nervous root so that the injured nerve is reconnected to the central nervous system through this new root, allowing patients who normally would have a permanent injury, fully or partially recover the movements, depending on the site. The nerve to be considered as a donor must fulfill a series of criteria such as being redundant, presenting synergistic characteristics to the receptor muscle and having size corresponding to the receptor nerve. This is only possible by a characteristic of the nervous system called neuroplasticity in which it is able to rearrange the somatotropic map, delegating new functions to different nerve fibers according to the patients's perception. This procedure classified as microsurgery must meet several criteria and its accomplishment should occur up to 6 months after the injury. RESULTS: These are variables, depending on the donor nerve and the substituted nerve, but more proximal regions that exert thick movements such as the shoulder have a better postoperative prognosis when compared to the musculature that require fine movements and accurate as elbow and wrist. CONCLUSION: This technique, despite having restrictions, is a means of restoring to patients important motor functions of the organism and also providing better quality of life to them, however, few professionals are skilled in the subject.
77.

CASE REPORT NEO ENDOTHELIUM IN INFECTED PROSTHESIS IN ARTERIOVENOUS FISTULA

Bárbara Cózaro Valentini, Otacílio de Camargo Junior, Rafael de Figueiredo Torres, Mariana Greccho Nunes, Rebecca Marcelino Ribeiro, Otacílio de Camargo Junior

Pontifícia Universidade Católica de Campinas

INTRODUCTION: During an aggression to the vascular endothelium an inflammatory reaction is generated with lymphocytes, monocytes, platelets and smooth muscle cells acting leading to endothelial cell dysfunction, which is characterized by a reduction in the bioavailability of endotheliumderived vasodilators and an increase, relative or absolute of vasoconstrictors, which impairs endothelium-dependent vasodilation. Autologous artery-venous fistula (AVF) is the ideal access for chronic renal patients, but when AVF cannot be performed, synthetic prostheses are used, which unfortunately provides a greater number of complications such as infection. These infections can cause arterial suture dehiscence, leading to difficult-to-control bleeding. In case of prosthesis infection, the goal of treatment is usually to remove the infected graft and restore vascular continuity. CASE REPORT: A 59-year-old male patient in dialytic treatment for chronic renal failure undercame upper limb AVF. Occlusion of AVF was detected and embolectomy was performed with thrombus removal from the prosthesis and a good postoperative thrill. Patient evolved with phlogistic signs and prosthesis infection. Surgery was performed to remove the prosthesis and, seventeen months later, the patient presented an abscess in the surgery's scar with purulent discharge, which was submitted to removal of infected segment of the prosthesis. DISCUSSION: Flow maintenance with distal bed irrigation is performed with the formation of a new endothelium. It is important after removing a synthetic prosthesis to place a saphenous vein flap to prevent rupture of the endothelium.

\section{ASSOCIATION OF EXTERNAL RADIOTHERAPY WITH HIGH DOSE RATE BRACHYTHERAPY IN PROSTATE CANCER TREATMENT}

Barbara Brunca, Leonardo Oliveira Reis

Pontifícia Universidade Católica de Campinas

Prostate cancer is the second most prevalent cancer among men in the world. Its treatment has a wide range of alternatives and variables, ranging from surveillance radiotherapy and / or brachytherapy and surgery. The present study aimed to identify predictive factors for biochemical recurrence and to evaluate the toxicity of treatment using the association of external rat therapy (RTe) with high brachytherapy dose rate (BATD) applied in the treatment of prostate cancer patients. The only variable that showed a statistically significant difference was the PSA nadir value It; 1 $\mathrm{ng} / \mathrm{ml}$. Three PSA stratifications showed statistical value in terms of biochemical recurrence: 1) PSA nadir $<1(p=0,018) ; 2)$ PSA nadir $(p=0,0009)$; 3) PSA pós $(p<0,0001)$. No other variables (age, initial PSA, dose used in the RTe, number of needles, number of positive biopsy fragments, blockade use Gleason score or TNM staging) was determinant for biochemical recurrence. The incidence of late grade 3 toxicity in the genitourinary tract was $0.6 \%$, and there were no cases of severe complications in the gastrointestinal tract intestinal.

Keywords: External Radiotherapy, High Dose Rate Brachytherapy, Prostate Cancer, Biochemical Recurrence, Toxicity, Efficacy.

\section{PERFUSION FLUID CONTAMINATION IN RELATION TO RECIPIENT SURVIVAL IN ORTHOTOPIC LIVER TRANSPLANTATION: RETROSPECTIVE ANALYSIS OF} THE LAST TEN YEARS.

Bárbara Regina Foster, Elaine Cristina de Ataíde

Universidade Estadual de Campinas - UNICAMP

INTRODUCTION: Liver-transplanted patients are more susceptible to the development of infections due to graft contamination, usually related to the preservation fluid employed in conserving the organ after its removal from the donor, and to the high serum concentration of immunosuppressants. Such patients are known to have higher mortality rates and worse prognoses. OBJECTIVES: Find an association between the contamination of the preservation fluid by bacteria and yeast and the systemic infection in patients after liver transplantation performed at HC-Unicamp from 2008 to 2017, correlating these results with their survival. PATIENTS AND METHODS: Preservation fluid cultures of patients that underwent liver transplantation at the State University of Campinas Clinical Hospital from January/2008 to December/2017 will be evaluated, correlating them to: biological fluids cultures (blood, urine and ascites), length of ICU stay, intubation time, opportunistic infections (pneumonia, urinary tract infection and sepsis), 
antibiotic regimen and its usage time, patients' SWAB at the immediate preoperative period, cold ischemia time and patient survival. In order to predict the highest risk of infection, acting preventively, it aims to contribute to the delicate postoperative management of liver transplantation.

Keywords: Preservation Fluid; Contamination; Orthotopic Liver Transplantation.

\section{ISOLATED ANEURYSM OF THE RIGHT COMMON ILIAC ARTERY}

Ornella Paola Gambarini, Otacílio de Camargo Júnior, Bárbara Cózaro Valentini, Rafael de Figueiredo Torres Caivano, Rebecca Marcelino Ribeiro, Otacílio de Camargo Junior

Pontifícia Universidade Católica de Campinas

BACKGROUND: The isolated iliac artery aneurysm is considered rare, with an incidence of approximately $1,5 \%$, most of the times associated to an abdominal aortic aneurysm. Necropsy studies show an incidence of $0,03 \%$. Its evolution is of growth until rupture, similar to the one of the abdominal aorta aneurysms, and the rupture can be to the retroperitoneal space of the pelvis or, more rarely, to the rectum or sigmoid colon. When they rupture, the have high rates of mortality, comparable to the ones of the abdominal aorta ruptured aneurysms. The external iliac artery generally isn't affected, the internal iliac artery is less frequently affected (around 10 to 30\%) and the common iliac is the most frequently affected (70 to 90\%), with a predominance on males. It is reported that the growth rate is slower in aneurysms smaller than $3 \mathrm{~cm}$, with 1,1 cm of growth per year, in comparison to $2,6 \mathrm{~cm}$ of growth per year in the aneurysms larger than $3 \mathrm{~cm}$, and with a rupture rate of 33 to $50 \%$ of the cases. The endovascular treatment is considered the best choice for this condition, once it has smaller mortality and complication rates when compared to open surgery. CASE REPORT: Male patient, 81 years old, diagnosed with an isolated aneurysm in the right common iliac artery, with $6,6 \mathrm{~cm}$ of extension and $3,85 \mathrm{~cm}$ of width in an computerized tomography, with symptom of pain in lumbar region. He was submitted to endovascular treatment with embolization of the right internal iliac artery with two $8 \mathrm{~mm}$ springs and one $10 \mathrm{~mm}$ spring. A Zenith Alpha Cook $22 \times 70$ prosthetic was placed in the abdominal aortic artery with $13 \times$ $77 \mathrm{~mm}$ of extension in left common iliac artery and $11 \times 125 \mathrm{~mm}$ of extension in right common iliac artery. The control angiography had no stenosis, no endoleaks and the arterial closure was made with perclose. The patient doesn't show complications after two months of follow-ups. DISCUSSION: The iliac artery aneurysm is, in the majority of times, asymptomatic and has the rupture as first symptom, requiring surgical treatment, preferably endovascular (depending on its caliber and association with the abdominal aorta). The endovascular surgery has been shown as very efficient to iliac artery aneurysms, having less surgical risks, specially because of its location in the pelvis, and a much inferior rate of complications in the postoperative, when compared to de open surgery of reconstruction.

81. THERMAL INJURY OF FEMORAL AND SUBCLAVIAN ARTERY: CASE REPORT Daniela Vallesquino Murayama, Otacílio de Camargo Junior, Mariana Greccho Nunes, Bárbara Cózaro Valentini, Rebecca Marcelino Ribeiro, Otacílio de Camargo Junior

Pontifícia Universidade Católica de Campinas

INTRODUCTION: Considered one of the most difficult aspects on the assistance of traumatized patients, the vascular trauma has been increasing due to the urban violence. The improve on the prehospital care and emergency transport has been allowing the assistance of patients who previously did not arrive alive at hospitals. Vascular injuries can presente immediate signals and symptoms like haemorrhage, ischemia or tumor, however, their absence does not represent absence of vascular injury. Lesions of the subclavian-axillary territory represent 1 to $2 \%$ of vascula traumas, and although not too usual, the injury in this region representes a mortality ranging from 10 to $35 \%$ and its morbidity is not negligible, with the lower limb injury being the one more frequently located. CASE REPORT: Case 1: Male patient, 45 years old, victim of two gunshot wound injuries on the left thigh. He was helped at another hospital, and at the time of the trauma had no symptoms other than pain at the wound site, being medicated and sutured. On the following day the patient was referred to the emergency room of the hospital with acute arterial occlusion in the left lower limb. On physical examination, the patient presented an ischemic limb with the absence of popliteal, anterior tibial and posterior tibial pulses. Ultrasound showed no arterial flow. He was submitted to resection of the thrombosed segment of superficial femoral artery and reversed saphenous vein interposition graft. Patient evolved in good general condition and with palpable distal pulses. Case 2: 19-year-old male patient checked in at the emergency room with gunshot injury, with entry hole in the posterior region of the right hemithorax, transfixing, and exit hole in the anterior region of the right hemithorax $2 \mathrm{~cm}$ below the clavicle in the medial third. On physical examination, the patient had ischemic right upper limb and no distal pulses. Doppler ultrasound examination showed three-phase flow in pre-injured subclavian artery and no post-lesion flow. The excision of the thrombosed subclavian artery segment was perfomed, followed by revascularization with saphenous vein segment, and the limb was reperfused with palpable pulses. DISCUSSION: The vascular injury can presente hemorrhagic, tumor or ischemic syndrome, however, we should consider the possibility of vascular injury even in the absence of these three syndromes when the lesion is in a vessel path.

Keywords: vascular injuries, ischemia

\section{PHLEGMASIA CERULEA DOLENS - FOUR CASES REPORT}

Rodrigo Otero Coelho, Bruna Madeleine da Silva Simplício Flôr, Daniel Olivatto Zanutto, Otacílio de Camargo Junior

Pontifícia Universidade Católica de Campinas

Phlegmasia Cerulea Dolens (PCD) is a rare syndrome resultant from a massive deep vein thrombosis in proximal vessels, characterized by the triad of severe pain, edema and cyanosis. The diagnosis is essentially clinical but can be supported using ultrasonography. The objective is a fast and effective treatment, because the risks of limb loss, massive embolism, compartmental syndrome and death are high. The article presents the report of four patients who were seen at the vascular surgery service of a tertiary hospital in the city of Campinas (SP). One patient was treated with fibrinolytic agents by systemic approach and two by endovascular approach, with one patient dying and the others with a favorable outcome. The absence of a universal protocol for the management of the pathology is a challenge, but the treatment objectives are clear: reestablish venous flow, valve function, preserve collateral circulation and reduce the risks of postthrombotic syndrome and the occurrence of new thrombi. The most commonly observed outcome is limb amputation, with differences in the literature regarding its prevalence. If gangrene is not established, a reversal of the condition is possible, except in circumstances of extensive capillary involvement. Death is also a probable outcome.

Keywords: Phlegmasia Cerulea Dolens; Thrombosis; Vascular Surgery; Case Report.

\section{COMPLICATION AFTER ARTERIOVENOUS FISTULA CONNECTION}

Carolina Ozawa Mançano, Rafael de Figueiredo Torres Caivano, Rebecca Marcelino Ribeiro, Mariana Greccho Nunes, Otacílio de Camargo Junior

Pontifícia Universidade Católica de Campinas

INTRODUCTION: Arteriovenous fistulas of the upper limbs are performed with prophylactic intent in cases of patients with borderline or therapeutic kidney for hemodialysis. THE high arterial flow through the venous segment results in arterialization and formation of single or multiple aneurysms in this vessel, so that they are definite accesses. Meantime, Repetitive punctures associated with aneurysmal dilation of AVF increase the risk of rupture from FAV. I am in this report a patient who presented to the physical examination pulsatile mass in the AVF region of approximately $5 \mathrm{~cm}$ with scars from previous surgeries. Same after undergoing resection of the aneurysmal mass and end-to-end anastomosis of brachial artery with good recovery, the patient returned to the operating room after 2 years with a pulsatile mass at the same site as the previous surgery and arteriomegaly of subclavian, axillary and brachial artery. MATERIAL AND METHOD: A 37 year-old female patient with chronic dialysis submitted to AVF in anothe service and also submitted to two other procedures post-AVF surgical procedures. Physical examination showed pulsatile mass in the AVF region of approximately $5 \mathrm{~cm}$ with scars from previous surgeries. Resected patient aneurysmal mass and end-to-terminal brachial artery anastomosis with good evolution postoperatively. RESULTS: Good evolution of palpable radial and ulnar pulses after surgery. of reconstruction. Two years after the surgical procedure, the patient returned to the service with presence of pulsatile mass at the same site as the previous surgery. At angiographic examination, Subclavian, axillary and brachial artery arteriomegaly. CONCLUSION: AVF for hemodialysis present a high incidence of complications, such as as aneurysms, infection, thrombosis and occlusion. Due to the risk of local infection, the use of Prostheses for arterial reconstruction should be avoided. AVF aneurysms may be treated with 
venous graft interposition or end-to-end reconstruction in the In the event, however, vigilance should be develop arterial dilation even after the aneurysm has been corrected.

\section{ANALYSIS OF RESIDENT PERFORMANCE IN A SIMULATED MULTIPLE VICTIM \\ INCIDENT}

David Cirigussi Rodrigues de Paula, Gustavo Pereira Fraga, Thiago Rodrigues Araújo Calderan

Universidade Estadual de Campinas - UNICAMP

INTRODUCTION: The number of people injured in traffic exceeded the 1.6 million mark in Brazil with an expense of approximately 3 billion reais per year. In some traumas there may be an imbalance between medical resource requirements and their availability, which characterizes a Multiple Victim Incident (IMV). This complex scenario needs trained and qualified professionals to deal with this occurrence. OBJECTIVES: This study aims to analyze the preparation of trauma surgery and emergency medicine residents to work in an IMV and to evaluate the performance during a simulated. METHODS: This is a cross-sectional study in which residents were assessed through a questionnaire about their preparedness in the face of a disaster and their performance analysis in a simulated IMV, through a checklist that evaluated the measures adopted in care. initial to traumatized, where A (Airway), B (Breathing), C (Circulation), D (Disability) and $E$ (Expousure). Descriptive analysis was performed by presenting frequency tables for categorical variables and position and dispersion measures for numerical variables. For comparison of proportions, Fisher's exact test was used. The significance level adopted for the statistical tests was $5 \%$. RESULTS: Regarding the analysis of the preparation of the seven residents who participated in the simulation, $71.4 \%$ were not involved in an IMV; $42.9 \%$ did not participate in a disaster simulation. Regarding performance, the hit rate was $91.39 \%$, but some procedures were not performed properly: in item A $26 \%$ did not use face mask with oxygen reservoir at $10 \mathrm{~L} /$ min correctly; in item B the oximetry was incorrectly evaluated in $20 \%$ of the cases; in item C not stopping external bleeding correctly occurred in $33.3 \%$; item D presented $26.7 \%$ of failures due to lack of Clasgow scale assessment; In item E promoting thermal insulation was performed incorrectly by $46.7 \%$ of residents. When comparing the resident profile, all residents who received formal training during their residency at IMV provided correct analgesia and $75 \%$ of those who did not missed ( $p=$ 0.1429 ).

Keywords: Multiple victims, Residents, Simulated.

85. COMPARATIVE STUDY BETWEEN MINIMALLY INVASIVE AND CONVENTIONAL TECHNIQUES FOR REPAIR OF CALCANEAL TENDON INJURY Guilherme Xavier de Lima Lutti, Letícia Francisco, Jorge Ramires Terrazas, Mariana Pereira Pinto, Cíntia Kelly Bittar Pontifícia Universidade Católica de Campinas

STUDY DESIGN: Retrospective comparative study. BACKGROUND: The calcaneal tendon is one that most commonly suffer from spontaneous ruptures, mainly occurring during recreational activities, especially in men between 30 and 40 years. However, $25 \%$ of these ruptures may occur in sedentary patients, usually due to chronic degeneration of the tendon. Different options and techniques for the repair of calcaneal tendon injuries are described in the literature. However, among the surgical treatments available lack a consensus on the treatment considered ideal. OBJECTIVE: Compare minimal invasive techniques using peroneus brevis tendon with conventional and open techniques, associated with reinforcement of semitendinosus tendon for the repair of calcaneal tendon injuries and clinical-functional results, degree of satisfaction and complications found. MATERIAL AND METHOD: A retrospective study including 43 patients with tendon calcaneal injuries. Dividing those submitted with minimally invasive groups and peroneus brevis tendon reinforcement from those submitted with open surgery with semitendinosus reinforcement. The variables evaluated were mean age, laterality, clinical-functional evaluation with American Orthopaedic Foot and Ankle Society (AOFAS) questionnaire and complications. RESULTS: Sixteen patients were treated with a minimally invasive procedure using peroneus brevis tendon graft, other 27 patients were treated with a semitendinosus tendon graft. $86 \%$ of them were male, $55,8 \%$ left laterality, $27,9 \%$ with degenerative lesions and $72,1 \%$ with traumatic injuries. The clinical-functional results were obtained after an average time of two years after the procedure. In the cases of open suture, there were three complications in the donor area (11.1\%), with a complaint of pain and insensitivity, three complications in receiving area (superficial skin necrosis, deep skin necrosis and graft and dehiscence of suture), totalizing six patients $(22.2 \%)$ with postoperative complications. In cases of minimally invasive suture, two complications occurred $(12.5 \%)$, but both were resolved prematurely. DISCUSSION: This study compared a traditional reconstruction of the calcaneal tendon using open technique associated with a tendinous graft, to a minimally invasive technique with graft, which aims to bring lower morbidity. Calcaneal tendon ruptures are common, specially in men, between the age of 40 and 50 years old. In this study the complications related to the skin manifested only in the open reconstruction group, compatible with information of the literature, with a case of deep skin infection, requiring debridement and posterior skin flap, and one case of superficial skin infection, with dehiscence of operative wound, besides a case of dehiscence no related with operative wound, without infection, but prolonging the period of rehabilitation. All these complications are frequent in patients submitted to open reconstruction. In addition, they were tabagists patients, who present a greater tendency of circulatory deficit in the skin, predisposing to greater local complications. The other three complications of the open reconstruction group were in the graft-donor area, in knee level, all with local sensitivity change. All patients in this group had complications completely resolved at the end of the treatment. In the minimally invasive reconstruction group there was no infection nor skin complication. Sural nerve lesion did not occurred in any of the groups studied; as well as re-rupture, demonstrating that tendinous graft may have contributed to minimize this complication, regardless of the technique used. The clinical-functional results obtained through the AOFAS postoperative questionnaire were superior in the group with minimally invasive reconstruction, with an average of 92 points against 88 points in the open reconstruction group, compatible with the literature, and the difference was mainly related to lower complications related to the operative wound in the minimally invasive group, while these caused a delay in the rehabilitation of some patients in the open reconstruction group. CONCLUSION: A minimally invasive technique using peroneus brevis tendon as graft is a good option for reconstruction of tendon calcaneal injuries. It offers an alternative to the open technique, due to a smaller number of complications related to operative wounds.

Keywords: Achilles tendon / surgery; Tendons injuries; Transplantation; Rupture.
This work is licensed under a Creative Commons Attribution 4.0 International License

ISSN 2076-6327

This journal is published by the University Library System, University of Pittsburgh as part of the Digital Publishing Program and is co-sponsored by the University of Pittsburgh Press. 